This is an electronic reprint of the original article. This reprint may differ from the original in pagination and typographic detail.

Author(s): Leonov, G. A.; Kuznetsov, Nikolay; Korzhemanova, N. A.; Kusakin, D. V.

Title: Lyapunov dimension formula for the global attractor of the Lorenz system

Year: $\quad 2016$

Version:

Please cite the original version:

Leonov, G. A., Kuznetsov, N., Korzhemanova, N. A., \& Kusakin, D. V. (2016). Lyapunov dimension formula for the global attractor of the Lorenz system. Communications in Nonlinear Science and Numerical Simulation, 41, 84-103.

https://doi.org/10.1016/j.cnsns.2016.04.032

All material supplied via JYX is protected by copyright and other intellectual property rights, and duplication or sale of all or part of any of the repository collections is not permitted, except that material may be duplicated by you for your research use or educational purposes in electronic or print form. You must obtain permission for any other use. Electronic or print copies may not be offered, whether for sale or otherwise to anyone who is not an authorised user. 


\section{Accepted Manuscript}

Lyapunov dimension formula for the global attractor of the Lorenz system

G.A. Leonov, N.V. Kuznetsov, N.A. Korzhemanova, D.V. Kusakin

PII: S1007-5704(16)30134-4

DOI: 10.1016/j.cnsns.2016.04.032

Reference: CNSNS 3853

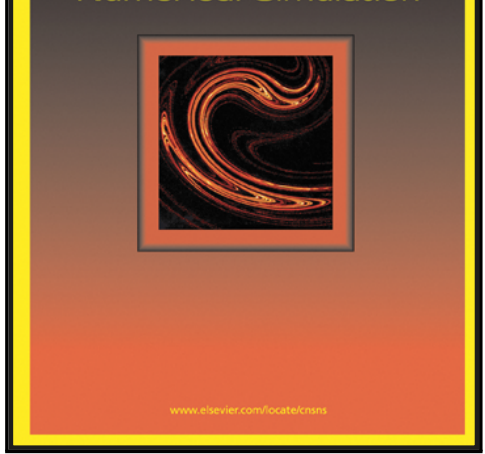

To appear in:

Communications in Nonlinear Science and Numerical Simulation

Received date:

28 August 2015

Revised date:

4 March 2016

Accepted date:

26 April 2016

Please cite this article as: G.A. Leonov, N.V. Kuznetsov, N.A. Korzhemanova, D.V. Kusakin, Lyapunov dimension formula for the global attractor of the Lorenz system, Communications in Nonlinear Science and Numerical Simulation (2016), doi: 10.1016/j.cnsns.2016.04.032

This is a PDF file of an unedited manuscript that has been accepted for publication. As a service to our customers we are providing this early version of the manuscript. The manuscript will undergo copyediting, typesetting, and review of the resulting proof before it is published in its final form. Please note that during the production process errors may be discovered which could affect the content, and all legal disclaimers that apply to the journal pertain. 


\section{Highlights}

- The exact formula for the global attractor of the Lorenz system is obtained.

- The validity of the formula for all classical values of parameters is proved.

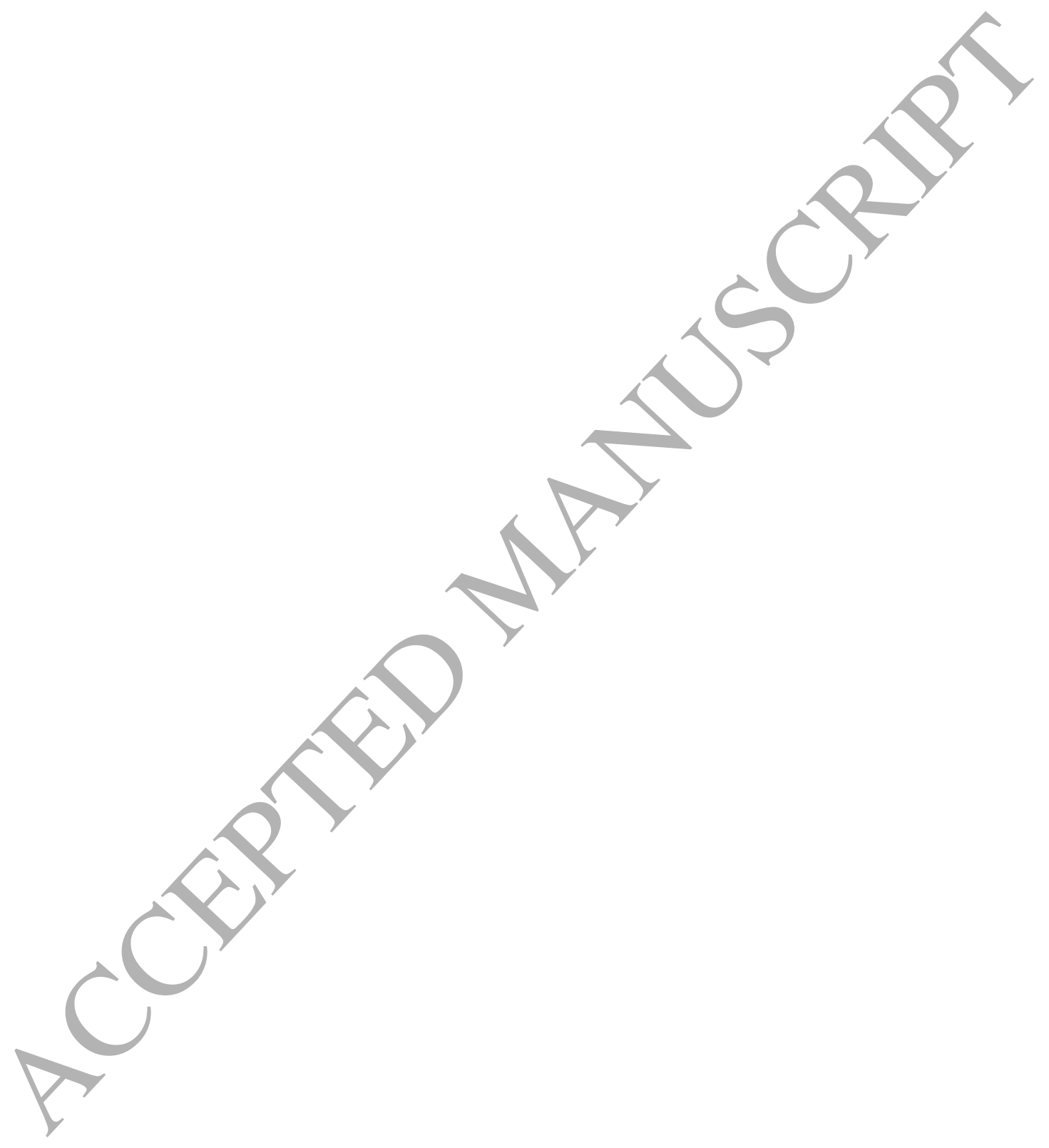




\title{
Lyapunov dimension formula for the global attractor of the Lorenz system
}

\author{
G.A. Leonov ${ }^{\mathrm{a}}$, N.V. Kuznetsove ${ }^{\mathrm{a}, \mathrm{b}}$, N.A. Korzhemanova ${ }^{\mathrm{a}}$, D.V. Kusakin ${ }^{\mathrm{a}}$ \\ ${ }^{a}$ Faculty of Mathematics and Mechanics, St. Petersburg State University, 198504 Peterhof, St. Petersburg, Russia \\ ${ }^{b}$ Department of Mathematical Information Technology, University of Jyväskylä, \\ 40014 Jyväskylä, Finland
}

\begin{abstract}
The exact Lyapunov dimension formula for the Lorenz system for a positive measure set of parameters, including classical values, has been analytically obtained first by G.A. Leonov in 2002 . Leonov used the construction technique of special Lyapunov-type functions, which was developed by him in 1991 year.

Later it was shown that the consideration of larger class of Lyapunovftype functions permits proving the validity of this formula for all parameters, of the system, such that all the equilibria of the system are hyperbolically unstable. In the present work it is proved the validity of the formula for Lyapunov dimension for a wider variety of parameters values including all parameters, which satisfy the classical physical limitations.
\end{abstract}

Keywords: Lorenz system, self-excited Lorenz attractor, Kaplan-Yorke dimension, Lyapunov dimension, Lyapunov exponents.

\section{Introduction}

The exact Lyapunov dimension formula for the Lorenz system for a positive measure set of parameters, including classical values, has been analytically obtained first by G.A.Leonov in 2002 [23]. In that work it was used the technique, of special Lyapunov-type functions, which had been created in 1991 [40] and was developed then in [43, 1]. Later in the works [42, 24, 37] it was shown that the consideration of a wider class of Lyapunov-type functions allows one to provide the validity of the formula for such parameters of the Lorenz system that all its equilibria are hyperbolically unstable.

In this study it is proved the validity of the obtained formula under classical restrictions on the parameters. One of the motivations for this woks is the numerical localization of chaotic attractor in the Lorenz system in the case of one unstable and two stable equilibria [53, 57].

\section{The Lorenz/system}

Consider the classical Lorenz system suggested in the original work of Edward Lorenz [46]:

$$
\left\{\begin{array}{l}
\dot{x}=\sigma(y-x) \\
\dot{y}=r x-y-x z \\
\dot{z}=-b z+x y .
\end{array}\right.
$$

Email address: Corresponding author: nkuznetsov239@gmail.com (N.V. Kuznetsov) 
Lorenz obtained his system as a truncated model of thermal convection in a fluid layer. The parameters of this system are positive:

$$
\sigma>0, r>0, b>0
$$

because of their physical meaning (e.g., $b=4\left(1+a^{2}\right)^{-1}$ is positive and bounded).

The active study of the Lorenz system gave rise to the appearance and subsequent consideration of various Lorenz-like systems (see, e.g. [3, 4, 47, 55]). A recent discussion on the equivalence of some Lorenz-like systems and the possibility of universal consideration of their behavior can be found, e.g. in $[27,30,28]$.

Since the system is dissipative and generates a dynamical system for $\forall t \geq 0$ (to verify this, it suffices to consider the Lyapunov function $V(x, y, z)=\frac{1}{2}\left(x^{2}+y^{2}+(z-r-\sigma)^{2}\right)$; see, e.g. [46, 1]), it possesses a global attractor (a bounded closed invariant set, which is globally attractive) [5, 1].

For the Lorenz system the following classical scenario of transition to chaos is known [53]. Suppose that $\sigma$ and $b$ are fixed (we use the classical parameters $\sigma=10, b=8 / 3$ ) and $r$ varies. Then, as $r$ increases, the phase space of the Lorenz system is subject to the following sequence of bifurcations. For $0<r<1$, the zero equilibrium $S_{0}$ is globally asymptotically stable. For $r>1$, the equilibrium $S_{0}$ is a saddle and a pair of symmetric equilibria/ $S_{1,2}$ appears. For $1<$ $r<r_{h} \approx 13.9$, the separatrices $\Gamma_{1,2}$ of equilibria $S_{0}$ are attracted to the equilibria $S_{1,2}$. For $r=r_{h} \approx 13.9$, the separatrices $\Gamma_{1,2}$ form two homoclinic trajectories of equilibria $S_{0}$ (homoclinic butterfly). For $r_{h}<r<r_{c} \approx 24.06$, the separatrices $\Gamma_{1}$ and $\Gamma_{2}$ tend to $S_{2}$ and $S_{1}$, respectively. For $r_{c}<r<r_{a} \approx 24.74$, the equilibria $S_{1,2}$ are stable and the separatrices $\Gamma_{1,2}$ may be attracted to a local chaotic attractor (see, e.g. $[53,57]$ ). The corresponding local attractor in Fig. 1 is selfexcited $^{1}$ with respect to the zero equilibrium and can be found using the standard computational procedure, i.e. by constructing a solution with initial data from a small neighborhood of the zero equilibrium, observing how it is attracted, and visualizing the attractor.

For $r>r_{a}$, the equilibria $S_{1,2}$ become unstable. The value $r=28$ corresponds to the classical self-excited (with respect to all equilibria) local attractor (see Fig. 2).

\section{Estimates of Lyapunov dimension via the Lyapunov direct method}

Consider an autonomous differential equation

$$
\dot{u}=f(u), \quad f: \mathbb{R}^{n} \rightarrow \mathbb{R}^{n}
$$

where $f$ is a continuously differentiable vector-function. Define by $u\left(t, u_{0}\right)$ a solution of $(2)$ such that $u\left(0, u_{0}\right)=u_{0}$, and consider the evolutionary operator $\varphi^{t}\left(u_{0}\right)=u\left(t, u_{0}\right)$. Assume the uniqueness and existence of solutions of $(2)$ for $t \in[0,+\infty)$. Then system (2) generates the dynamical

\footnotetext{
${ }^{1}$ An oscillation can generally be easily numerically localized if the initial data from its open neighborhood in the phase space (with the exception of a minor set of points) lead to a long-term behavior that approaches the oscillation. Therefore, from a computational perspective, it is natural to suggest the following classification of attractors $[21,34,35,26]$, which is based on the simplicity of finding their basins of attraction in the phase space: An attractor is called a self-excited attractor if its basin of attraction intersects with any open neighborhood of an equilibrium, otherwise it is called a hidden attractor $[21,34,35,26,17]$. Up to now in such Lorenz-like systems as Lorenz, Chen, Lu, and Tigan systems only self-excited chaotic attractors were found. In such Lorenz-like systems as Glukhovsky-Dolghansky and Rabinovich systems both self-excited and hidden attractors can be found [20, 31, 32] . Recent examples of hidden attractors can be found in The European Physical Journal Special Topics: Multistability: Uncovering Hidden Attractors, 2015 (see [51, 2, 15, 58, 49, 50, 14, 45, 13, 54, 48, 56, 52]). Note that while coexisting self-excited attractors can be found by the standard computational procedure, there is no regular way to predict the existence or coexistence of hidden attractors.
} 


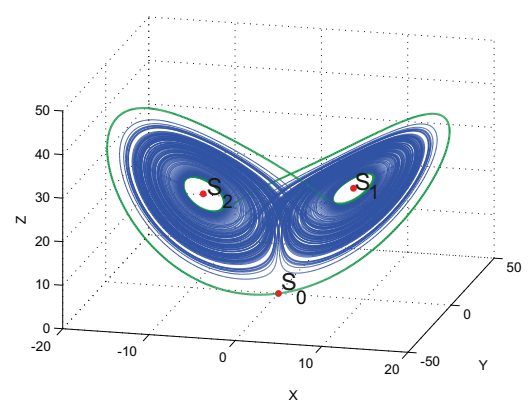

(a)

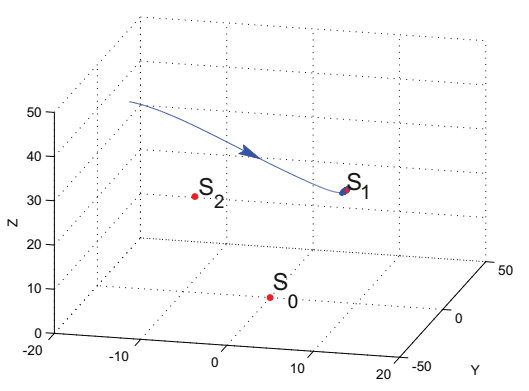

(b)

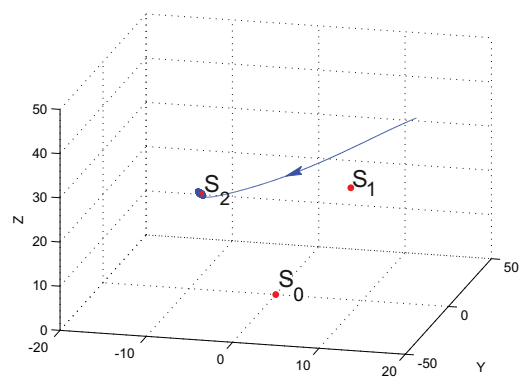

(c)

Figure 1: (a) Numerical visualization of a self-excited Lorenz attractor by using a trajectory with an initial point taken in the vicinity of the equilibrium $S_{0}$. (b), (c) Trajectories with the initial data $(\mp 16.2899, \mp 0.0601,42.1214)$ tend to equilibria $S_{2,1}$. Parameters: $r=24.5, \sigma=10, b=8 / 3$.

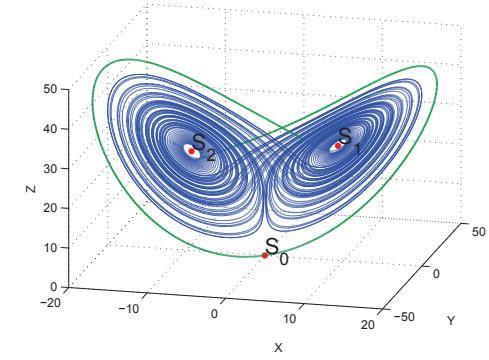

(a) Initial data near the equilibrium $S_{0}$

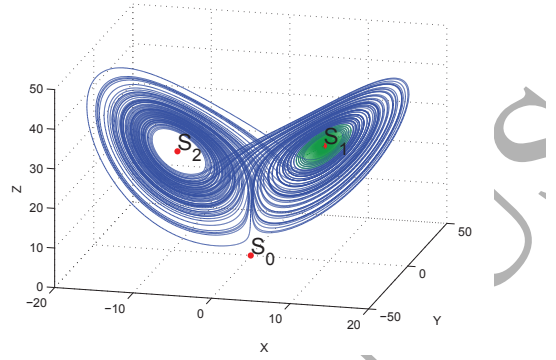

(b) Initial data near the equilibrium $S_{1}$

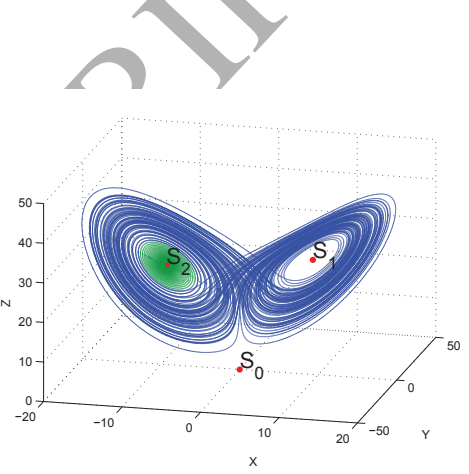

(c) Initial data near the equilibrium $S_{2}$

Figure 2: Numerical visualization of the classical self-excited local attractor in the Lorenz system by using the trajectories that start in small neighborhoods of the unstable equilibria $S_{0,1,2}$. Here the separation of the trajectory into transition process (green) and approximation of attractor (blue) are rough.

system $\left\{\varphi^{t}\right\}_{t \geq 0}$. Let a nonempty set $K \subset \mathbb{R}^{n}$ be invariant with respect to $\left\{\varphi^{t}\right\}_{t \geq 0}$, i.e. $\varphi^{t}(K)=K$ for all $t>0$. Consider the linearization of system (2) along the solution $\varphi^{t}(u)$ :

$$
\dot{v}=J\left(\varphi^{t}(u)\right) v, \quad J(u)=D f(u),
$$

where $J(u)$ is the $n \times n$ Jacobian matrix, the elements of which are continuous functions of $u$. Suppose that det $J(u) \neq 0 \quad \forall u \in \mathbb{R}^{n}$. Consider a fundamental matrix of linearized system (3) $D \varphi^{t}(u)$ such that $D \varphi^{0}(u)=I$, where $I$ is a unit $n \times n$ matrix. Let $\sigma_{i}(t, u)=\sigma_{i}\left(D \varphi^{t}(u)\right)$, $i=1,2, . . n$, be the singular values of $D \varphi^{t}(u)$ (i.e. $\sigma_{i}(t, u)>0$ and $\sigma_{i}(t, u)^{2}$ are the eigenvalues of the symmetric matrix $D \varphi^{t}(u)^{*} D \varphi^{t}(u)$ with respect to their algebraic multiplicity $)^{2}$ ordered so that $\sigma_{1}(t, u) \geq \ldots \geq \sigma_{n}(t, u)>0$ for any $u$ and $t \geq 0$. The singular value function of order $d \in[0, n]$ at $u \in U$ is defined as

$$
\omega_{d}\left(D \varphi^{t}(u)\right)=\sigma_{1}(t, u) \cdots \sigma_{\lfloor d\rfloor}(t, u) \sigma_{\lfloor d\rfloor+1}(t, u)^{d-\lfloor d\rfloor}, \quad d \in[0, n),
$$

where $\lfloor d\rfloor$ is the largest integer less or equal to $d$ and $\omega_{n}\left(D \varphi^{t}(u)\right)=\sigma_{1}(t, u) \cdots \sigma_{n}(t, u)$.

The concept of the Lyapunov dimension was suggested in the seminal paper by Kaplan and Yorke [16] and later it was developed in a number of papers. The following definition is inspirited by Douady-Oesterlé [8]. The local Lyapunov dimension of the map $\varphi^{t}$ at the point $u \in \mathbb{R}^{n}$ is

$2 *$ denotes matrix transposition. 
defined as [18]

$$
\operatorname{dim}_{\mathrm{L}}\left(\varphi^{t}, u\right)=\max \left\{d \in[0, n]: \omega_{d}\left(D \varphi^{t}(u)\right) \geq 1\right\}
$$

and the Lyapunov dimension of the map $\varphi^{t}$ with respect to invariant set $K$ is defined as

$$
\operatorname{dim}_{\mathrm{L}}\left(\varphi^{t}, K\right)=\sup _{u \in K} \operatorname{dim}_{\mathrm{L}}\left(\varphi^{t}, u\right)=\sup _{u \in K} \max \left\{d \in[0, n]: \omega_{d}\left(D \varphi^{t}(u)\right) \geq 1\right\} .
$$

In the paper [8] Douady and Oesterlé it is rigorously proved that the Lyapunov dimension of the map $\varphi^{t}$ with respect to the compact invariant set $K$ is an upper estimate of the Hausdorff dimension of the set $K$. Thus we have

$$
\operatorname{dim}_{\mathrm{H}} K \leq \inf _{t \geq 0} \operatorname{dim}_{\mathrm{L}}\left(\varphi^{t}, K\right)
$$

Here $\inf _{t \geq 0} \operatorname{dim}_{\mathrm{L}}\left(\varphi^{t}, K\right)$ is called the Lyapunov dimension of dynamical system $\left\{\varphi^{t}\right\}_{t \geq 0}$ with respect to invariant set $K$. For computations it is important that (see, e.g. [18])

$$
\inf _{t \geq 0} \operatorname{dim}_{\mathrm{L}}\left(\varphi^{t}, K\right)=\liminf _{t \rightarrow+\infty} \operatorname{dim}_{\mathrm{L}}\left(\varphi^{t}, K\right) .
$$

Consider the finite-time Lyapunov exponents at the point $u$ :

$$
\mathrm{LE}_{i}(t, u)=\frac{1}{t} \ln \sigma_{i}(t, u), i=1,2, \ldots, n \quad t \geqslant 0
$$

If $n>\operatorname{dim}_{\mathrm{L}}\left(\varphi^{t}, u\right)>1$, then for $j(t, u)=\left\lfloor\operatorname{dim}_{\mathrm{L}}\left(\varphi^{t}, u\right)\right\rfloor$ and $s(t, u)=\operatorname{dim}_{\mathrm{L}}\left(\varphi^{t}, u\right)-\left\lfloor\operatorname{dim}_{\mathrm{L}}\left(\varphi^{t}, u\right)\right\rfloor$ we have $0=\frac{1}{t} \ln \left(\omega_{j(t, u)+s(t, u)}\left(D \varphi^{t}(u)\right)\right)=\sum_{i=1}^{j(t, u)} \operatorname{LE}_{i}(t, u)+s(t, u) \operatorname{LE}_{j(t, u)+1}(t, u)$. The representation

$$
\operatorname{dim}_{\mathrm{L}}\left(\varphi^{t}, u\right)=j(t, u)+\frac{\mathrm{LE}_{1}(t, u)+\cdots+\mathrm{LE}_{j(t, u)}(t, u)}{\left|\mathrm{LE}_{j(t, u)+1}(t, u)\right|}
$$

corresponds to the Kaplan-Yorke formula [16] with respect to the finite-time Lyapunov exponents ${ }^{3}$. Remark that here $j(t, u)=\max \left\{m: \sum_{i=1}^{m} \operatorname{LE}_{i}(t, u) \geq 0\right\}$ and $\operatorname{LE}_{j(t, u)+1}(t, u)<0$ for $j(t, u)<n$.

Consider an effective analytical method, proposed by G.A. Leonov in 1991 [40] (see also [43, 23, $1,41,42,27,32])$. The advantage of this approach is that it allows one to estimate the Lyapunov dimension of invariant sets without localization of the set in the phase space and, in many cases, to get effectively exact Lyapunov dimension formula [23, 38, 37, 27, 30, 25, 29, 33]. Consider a nonsingular $n \times n$ matrix $S$. Let $\lambda_{i}\left(u_{0}, S\right), i=1,2, \ldots, n$, be the eigenvalues of the symmetrized Jacobian matrix

$$
\frac{1}{2}\left(S J\left(u\left(t, u_{0}\right)\right) S^{-1}+\left(S J\left(u\left(t, u_{0}\right)\right) S^{-1}\right)^{*}\right)
$$

ordered so that $\lambda_{1}\left(u_{0}, S\right) \geq \cdots \geq \lambda_{n}\left(u_{0}, S\right)$ for any $u_{0}$.

Theorem 1. Let $d=(j+s) \in[1, n]$, where integer $j=\lfloor d\rfloor \in\{1, \ldots, n\}$ and real $s=(d-\lfloor d\rfloor) \in$ $[0,1)$. If there exist a differentiable scalar function $V: U \subseteq \mathbb{R}^{n} \rightarrow \mathbb{R}^{1}$ and a nonsingular $n \times n$ matrix $S$ such that

$$
\sup _{u \in K}\left(\lambda_{1}(u, S)+\cdots+\lambda_{j}(u, S)+s \lambda_{j+1}(u, S)+\dot{V}(u)\right)<0,
$$

\footnotetext{
${ }^{3}$ While in the computations we can consider only finite time $t$ and evolutionary operator $\varphi^{t}(u)$, from a theoretical point of view, it can be considered the limit values of finite-time Lyapunov exponents (LEs). For example, KaplanYorke formula with respect to LEs is considered in $[6,12]$. Relying on ergodicity, the LEs and Lyapunov dimension of attractor are often computed along one trajectory, which is attracted or belongs to the attractor. But, in general, one has to consider a grid of points on $K$ and find the maximum of corresponding local Lyapunov dimensions (see, e.g. [22, 32]).
} 
where $\dot{V}(u)=(\operatorname{grad}(V))^{*} f(u)$, then

$$
\operatorname{dim}_{\mathrm{H}} K \leq \operatorname{dim}_{\mathrm{L}}\left(\left\{\varphi^{t}\right\}_{t \geq 0}, K\right) \leq \operatorname{dim}_{\mathrm{L}}\left(\varphi^{T}, K\right) \leq j+s
$$

for all sufficiently large $T>0$.

A proof of this theorem, based on the invariance of Lyapunov dimension with respect to diffeomorphisms, is given in [18] (see also [25, 19]).

Corollary 1. If for $d=j+s$ defined by Theorems 1 at an equilibrium point $u_{e q}^{c r} \equiv \varphi^{t}\left(u_{e q}^{c r}\right)$ the relation

$$
\operatorname{dim}_{\mathrm{L}}\left(\left\{\varphi^{t}\right\}_{t \geq 0}, u_{e q}\right)=j+s
$$

holds, then for any invariant set $K \ni u_{e q}^{c r}$ we get the formula of exact Lyapunov dimension

$$
\operatorname{dim}_{\mathrm{L}}\left(\left\{\varphi^{t}\right\}_{t \geq 0}, K\right)=\operatorname{dim}_{\mathrm{L}}\left(\left\{\varphi^{t}\right\}_{t \geq 0}, u_{e q}^{c r}\right)=j+s .
$$

For the study of continuous time dynamical system in $\mathbb{R}^{3}$ the following result is useful. Consider a certain open set $K_{\varepsilon} \subset \mathbb{R}^{n}$, which is diffeomorphic to a ball, whose boundary $\partial \overline{K_{\varepsilon}}$ is transversal to the vectors $f(u), u \in \partial \overline{K_{\varepsilon}}$. Let the set $K_{\varepsilon}$ be a positively invariant for the solutions of system $(2)$.

Theorem 2. (see [40, 41]] Suppose, a continuously differentiable function $V(u)$ and a nondegenerate matrix $S$ exist such that

$$
\lambda_{1}(u, S)+\lambda_{2}(u, S)+\dot{V}(u)<0, \forall u \in K_{\varepsilon} .
$$

Then any solution of system (2) with the initial data $u_{0} \in K_{\varepsilon}$ tends to the stationary set as $t \rightarrow+\infty$.

\section{Exact Lyapunov dimension of the global Lorenz attractor}

Further we will consider a bounded set $K$, which is invariant with respect to the Lorenz system, and use the compact notation $\operatorname{dim}_{\mathrm{L}} K$ for the Lyapunov dimension of the Lorenz system instead of $\liminf _{t \rightarrow+\infty} \operatorname{dim}_{\mathrm{L}}\left(\varphi^{t}, K\right)$.

By Theorems 1 and 2 for the Lorenz system we can obtain the following result.

Theorem 3. Let $\Omega=\Omega(\sigma, b, r)$ be the domain in three-dimensional space of parameters $(\sigma, b, r)$, where the following two inequalities

$$
\begin{gathered}
r-1>0 \\
r-1 \geq \frac{b(b+\sigma-1)^{2}-4 \sigma\left(b+\sigma b-b^{2}\right)}{3 \sigma^{2}}
\end{gathered}
$$

are hold and one of the following two conditions (a) and (b) is satisfied:

(a)

(b) let the equation

$$
\sigma^{2}(r-1)(b-4) \leq 4 \sigma\left(\sigma b+b-b^{2}\right)-b(b+\sigma-1)^{2} ;
$$

$$
\begin{array}{r}
(2 \sigma-b+\gamma)^{2}\left(b(b+\sigma-1)^{2}-4 \sigma\left(\sigma b+b-b^{2}\right)+\sigma^{2}(r-1)(b-4)\right)+ \\
+4 b \gamma(\sigma+1)\left(b(b+\sigma-1)^{2}-4 \sigma\left(\sigma b+b-b^{2}\right)-3 \sigma^{2}(r-1)\right)=0
\end{array}
$$

have two distinct real roots $\gamma^{(I I)}>\gamma^{(I)}$ and

$$
\left\{\begin{array}{l}
\sigma^{2}(r-1)(b-4)>4 \sigma\left(\sigma b+b-b^{2}\right)-b(b+\sigma-1)^{2} \\
\gamma^{(I I)}>0
\end{array}\right.
$$


1. If

$$
\begin{array}{r}
2 \sigma-b<(\sigma+1)\left(\sigma r^{-1}+1\right) \max _{\lambda \in\left[0, \lambda_{0}\right]} \frac{\lambda(b-\lambda)}{(\sqrt{r \sigma}-\sqrt{(\sigma-\lambda)(1-\lambda)})^{2}}, \\
\lambda_{0}=\min \{1, b, \sigma\}, \quad r-1>0
\end{array} \begin{aligned}
& \text { or }(\sigma, b, r) \in \Omega \text { and } \quad(b-\sigma)(b-1)<\sigma r<(b+1)(b+\sigma),
\end{aligned}
$$

then any bounded solution of system (1) tends to a certain equilibrium as $t \rightarrow+\infty$ and

$$
\operatorname{dim}_{\mathrm{L}} K=0
$$

2. If $(\sigma, b, r) \in \Omega$ and

$$
\sigma r>(b+1)(b+\sigma)
$$

then

$$
\operatorname{dim}_{\mathrm{L}} K \leq 3-\frac{2(\sigma+b+1)}{\sigma+1+\sqrt{(\sigma-1)^{2}+4 \sigma r}} .
$$

From numerical experiments (see, e.g. $[12,7]$ ) it is known that the Lyapunov dimension of the zero equilibrium is an upper bound for the Lyapunov dimension of any invariant compact set of the Lorenz system. This fact is also known [36] as the Eden conjecture on the Lorenz system (see, e.g. [10, p.411,Question 3.],[9, p.98, Question 2.], [11]).

Lemma 1. (see, e.g. [11, 23]) If for the parameters of system (1) inequality (17) is valid, then

$$
\operatorname{dim}_{L}(0,0,0)=3-\frac{2(\sigma+b+1)}{\sigma+1+\sqrt{(\sigma-1)^{2}+4 r \sigma}} .
$$

Since a global attractor contains all equilibria of the considered system and estimation (18) coincide with (19), by Corollary 1 we get exact Lyapunov dimension (Lyapunov dimension formula) of the global Lorenz attractor (or any invariant set, which contains the zero equilibrium).

Theorem 4. Let $K$ be a global attractor of classical Lorenz system (1) and conditions (10)-(14) of Theorem 3 be valid.

In this case if

then

$$
\sigma r>(b+1)(b+\sigma)
$$

$$
\operatorname{dim}_{\mathrm{L}} K=3-\frac{2(\sigma+b+1)}{\sigma+1+\sqrt{(\sigma-1)^{2}+4 \sigma r}} .
$$

Corollary 2. If all three equilibria are hyperbolic, then the conditions of Theorem 4 are satisfied.

The numerical check of this Corollary is shown in Fig. 3: L2 is the domain, where all three equilibria are hyperbolic, and $L 1$ is the domain, where the conditions of Theorem 4 are satisfied. For example, for the standard parameters $\sigma=10$ and $b=\frac{8}{3}$ formula (20) is valid for $r>\frac{209}{45}$. Thus, the result, obtained in [37] is a particular case of Theorem 3.

Corollary 3. For $1<b \leq 2$ and $r>1$ the conditions of Theorem 3 are satisfied.

Thus, the result, obtained in [11], is a particular case of Theorem 3.

Taking into account the physical meaning of parameters, let us consider $0<b<4$. In this case the following Corollary can be proved 

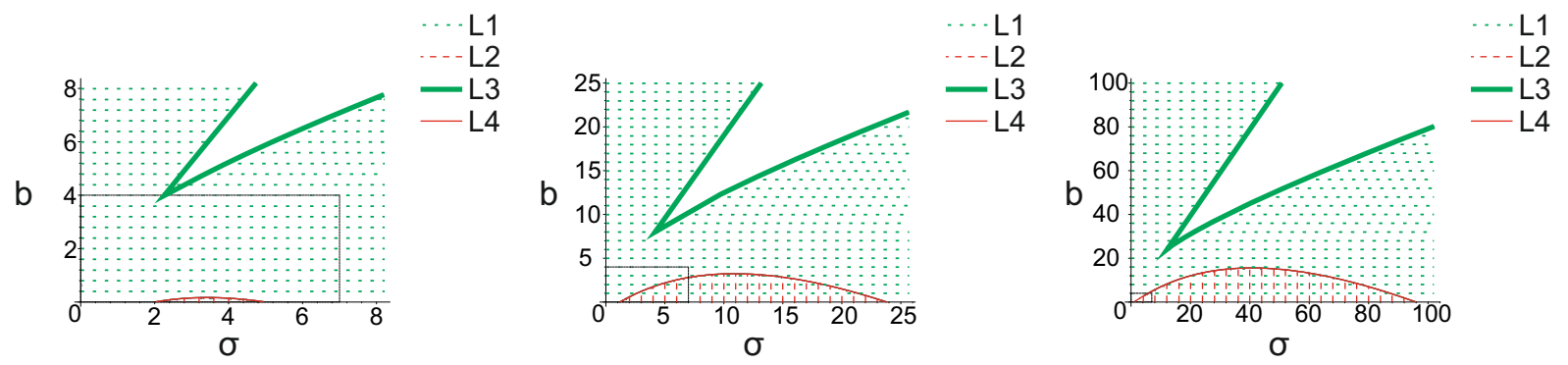

Figure 3: Domains of parameters $(\sigma, b)$ for $r=10,28,100$ : the conditions of Theorem 3 hold — shaded by $L 1$, the corresponding boundary is $L 3$; three equilibria are hyperbolic — shaded by $L 2$, the corresponding boundary is $L 4$.

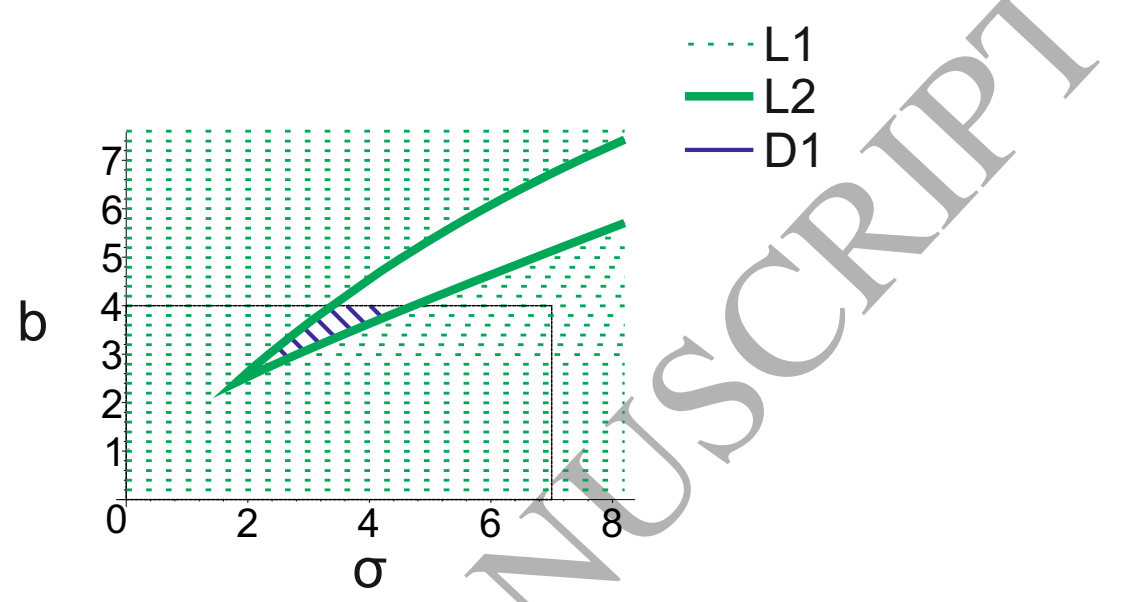

Figure 4: Domain of parameter $(\sigma, b)$ for $r=3$ : the conditions of Theorem 3 are valid - shaded by $L 1$, the corresponding boundary is $L 2$; the conditions of Theorem 3 are not valid for $0<b<4-D 1$.

Corollary 4. For all $r>1, \sigma>7$, and $0<b<4$ the conditions of Theorem 3 are satisfied.

Thus, for each $r>1$ it remains to check numerically the behavior of system with the parameters from the bounded domain $[0<b<4,0<\sigma \leq 7]$ (see, e.g. domain D1 in Fig. 4). We consider an increasing sequence $r_{k}>1$ and numerically integrate trajectories with initial data from the bounded absorbing set [44]. Our numerical simulation shows the lack of chaotic attractor and, thus, in the considered domain the Lyapunov dimension formula (20) is valid.

\section{Proofs of the statements}

\subsection{Proof of Theorem 3}

For the proof we use Theorems 1, 2 and the approaches to the choice of $S$ and $V$ from [36, 23, 37].

Consider a solution of $(1) u\left(t, u_{0}\right)=\left(x\left(t, x_{0}\right), y\left(t, y_{0}\right), z\left(t, z_{0}\right)\right)$.

I. For system (1) the matrix $J$ has the following form

$$
J=\left(\begin{array}{ccc}
-\sigma & \sigma & 0 \\
r-z & -1 & -x \\
y & x & -b
\end{array}\right)
$$

Following [23], for

$$
\sigma r+(\sigma-b)(b-1)>0
$$


we introduce a matrix

$$
S=\left(\begin{array}{ccc}
-\rho^{-1} & 0 & 0 \\
-\frac{b-1}{\sigma} & 1 & 0 \\
0 & 0 & 1
\end{array}\right)
$$

where

$$
\rho=\frac{\sigma}{\sqrt{\sigma r+(\sigma-b)(b-1)}}
$$

Then

$$
S J S^{-1}=\left(\begin{array}{ccc}
b-\sigma-1 & -\frac{\sigma}{\rho} & 0 \\
-\frac{\sigma}{\rho}+\rho z & -b & -x \\
-\rho\left(y+\frac{b-1}{\sigma} x\right) & x & -b
\end{array}\right) .
$$

Consider the characteristic polynomial of the matrix $\frac{1}{2}\left(S J S^{-1}+\left(S J S^{-1}\right)^{*}\right)$ :

$$
(\lambda+b)\left[\lambda^{2}+(\sigma+1) \lambda+b(\sigma-b+1)-\left(\frac{\sigma}{\rho}-\frac{\rho z}{2}\right)^{2}-\left(\frac{\rho(b-1)}{2 \sigma} x+\frac{\rho}{2} y\right)^{2}\right] .
$$

Such a choice of the matrix $S$ allows one to simplify the expression for the eigenvalues. The corresponding eigenvalues $\lambda_{i}=\lambda_{i}(x, y, z, S), i=1,2,3$, are the following numbers

$$
\lambda_{2}=-b, \lambda_{1,3}=-\frac{\sigma+1}{2} \pm \frac{1}{2}\left[(\sigma-2 b+1)^{2}+\left(\frac{2 \sigma}{\rho}-\rho z\right)^{2}+\rho^{2}\left(y+\frac{b-1}{\sigma} x\right)^{2}\right]^{\frac{1}{2}} .
$$

II. We shall prove that $\lambda_{1} \geq \lambda_{2} \geq \lambda_{3}, \forall x, y, z$ :

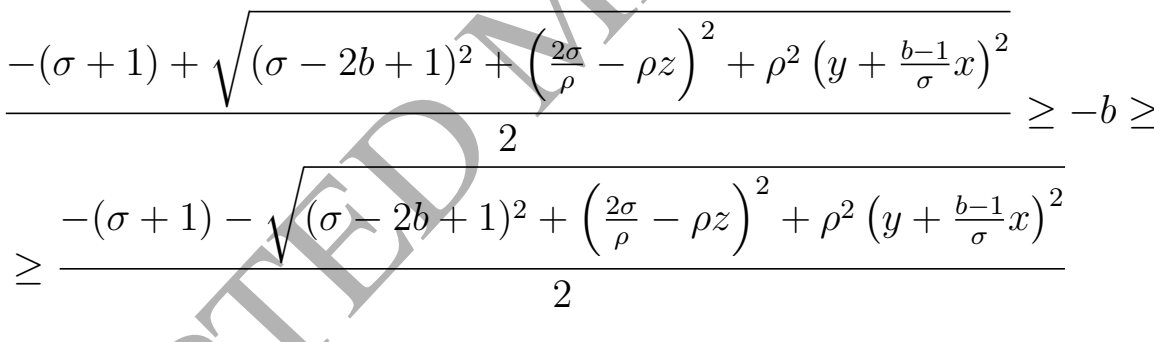

$$
\begin{aligned}
& \Leftrightarrow
\end{aligned}
$$

$$
\begin{aligned}
& \sqrt{(\sigma-2 b+1)^{2}+\left(\frac{2 \sigma}{\rho}-\rho z\right)^{2}+\rho^{2}\left(y+\frac{b-1}{\sigma} x\right)^{2}} \geq \sigma-2 b+1 \geq \\
& 7-\sqrt{(\sigma-2 b+1)^{2}+\left(\frac{2 \sigma}{\rho}-\rho z\right)^{2}+\rho^{2}\left(y+\frac{b-1}{\sigma} x\right)^{2}}
\end{aligned}
$$

$$
\begin{gathered}
\Leftrightarrow \\
\sqrt{(\sigma-2 b+1)^{2}+\left(\frac{2 \sigma}{\rho}-\rho z\right)^{2}+\rho^{2}\left(y+\frac{b-1}{\sigma} x\right)^{2}} \geq|\sigma-2 b+1| \\
\left(\frac{2 \sigma}{\rho}-\rho z\right)^{2}+\rho^{2}\left(y+\frac{b-1}{\sigma} x\right)^{2} \geq 0 .
\end{gathered}
$$

In the left-side there is a sum of two total squares, i.e. the inequality is always satisfied. Thus, $\lambda_{1} \geq \lambda_{2} \geq \lambda_{3}$ for all $x, y, z$. 
III. To apply Theorem 1 and Theorem 2, we transform the relation $2\left(\lambda_{1}+\lambda_{2}+s \lambda_{3}\right)$ with $s \in[0,1):$

$$
\begin{aligned}
& 2\left(\lambda_{1}+\lambda_{2}+s \lambda_{3}\right)=-(\sigma+2 b+1)-s(\sigma+1)+ \\
& +(1-s)\left[(\sigma-2 b+1)^{2}+\left(\frac{2 \sigma}{\rho}-\rho z\right)^{2}+\rho^{2}\left(y+\frac{b-1}{\sigma} x\right)^{2}\right]^{\frac{1}{2}}= \\
& -(\sigma+2 b+1)-s(\sigma+1)+ \\
& +(1-s)\left[(\sigma-2 b+1)^{2}+\frac{4 \sigma^{2}}{\rho^{2}}-4 \sigma z+\rho^{2} z^{2}+\rho^{2}\left(y+\frac{b-1}{\sigma} x\right)^{2}\right]^{\frac{1}{2}}= \\
& =-(\sigma+2 b+1)-s(\sigma+1)+ \\
& +(1-s)\left[(\sigma-1)^{2}+4 \sigma r-4 \sigma z+\rho^{2} z^{2}+\rho^{2}\left(y+\frac{b-1}{\sigma} x\right)^{2}\right]^{\frac{1}{2}}
\end{aligned}
$$

Next we use the inequality

$$
\sqrt{k+l} \leq \sqrt{k}+\frac{l}{2 \sqrt{k}}
$$

for $k+l>0, k>0$. Consider

$$
k+l=(\sigma-1)^{2}+4 \sigma r-4 \sigma z+\rho^{2} z^{2}+\rho^{2}\left(y+\frac{b-1}{\sigma} x\right)^{2}
$$

and

$$
k=(\sigma-1)^{2}+4 \sigma r .
$$

According to (23), the following equation

$$
\begin{aligned}
& k+l=(\sigma-1)^{2}+4 \sigma r-4 \sigma z+\rho^{2} z^{2}+\rho^{2}\left(y+\frac{b-1}{\sigma} x\right)^{2}= \\
& =(\sigma-2 b+1)^{2}+\left(\frac{2 \sigma}{\rho}-\rho z\right)^{2}+\rho^{2}\left(y+\frac{b-1}{\sigma} x\right)^{2} \geq 0
\end{aligned}
$$

is valid. By the condition of Theorem 3 we have

$$
r \sigma+(\sigma-b)(b-1)>0 \Leftrightarrow r \sigma>-\sigma b+\sigma+b^{2}-b .
$$

Then

$$
k=(\sigma-1)^{2}+4 \sigma r>(\sigma-1)^{2}-4 \sigma b+4 \sigma+4 b^{2}-4 b=(\sigma-2 b+1)^{2} \geq 0 .
$$

Applying inequality (24) with $k=(\sigma-1)^{2}+4 \sigma r$ and $l=-4 \sigma z+\rho^{2} z^{2}+\rho^{2}\left(y+\frac{b-1}{\sigma} x\right)^{2}$ to relation (23), we obtain

$$
\begin{aligned}
& 2\left(\lambda_{1}+\lambda_{2}+s \lambda_{3}\right) \leq-(\sigma+2 b+1)-s(\sigma+1)+(1-s)\left[(\sigma-1)^{2}+4 \sigma r\right]^{\frac{1}{2}}+ \\
& +\frac{2(1-s)}{\left[(\sigma-1)^{2}+4 \sigma r\right]^{\frac{1}{2}}}\left[-\sigma z+\frac{\rho^{2} z^{2}}{4}+\frac{\rho^{2}}{4}\left(y+\frac{b-1}{\sigma} x\right)^{2}\right] .
\end{aligned}
$$

Introduce the function

$$
V(x, y, z)=\frac{(1-s) \theta(x, y, z)}{\left[(\sigma-1)^{2}+4 \sigma r\right]^{\frac{1}{2}}}
$$


where

$$
\theta(x, y, z)=\gamma_{4} x^{2}+\left(-\sigma \gamma_{1}+\gamma_{3}\right) y^{2}+\gamma_{3} z^{2}+\frac{1}{4 \sigma} \gamma_{1} x^{4}-\gamma_{1} x^{2} z-\gamma_{1} \gamma_{2} x y-\frac{\sigma}{b} z .
$$

We choose the running parameters $\gamma_{1}, \gamma_{2}, \gamma_{3}, \gamma_{4}$ in such a way that

$$
R:=-\sigma z+\frac{\rho^{2} z^{2}}{4}+\frac{\rho^{2}}{4}\left(y+\frac{b-1}{\sigma} x\right)^{2}+\dot{\theta} \leq 0 \quad \forall x, y, z,
$$

where $\dot{\theta}(x)$ is a derivative with respect to system (1). Then, using (25), we obtain

$$
2\left(\lambda_{1}+\lambda_{2}+s \lambda_{3}\right)+2 \dot{V} \leq-(\sigma+2 b+1)-s(\sigma+1)+(1-s)\left[(\sigma-1)^{2}+4 \sigma r\right]^{\frac{1}{2}},
$$

i.e. we estimated $2\left(\lambda_{1}+\lambda_{2}+s \lambda_{3}\right)+2 \dot{V}$ using the relation, which depends on the parameters of system (1) and is independent of $x, y, z$.

IV. We perform the analysis of $R$, choosing the running parameters $\gamma_{1}, \gamma_{2}, \gamma_{3}, \gamma_{4}$ in such a way that (28) is valid. Taking into account (27), we obtain

$$
\begin{gathered}
R=-\gamma_{1} x^{4}+\left(\gamma_{1} \gamma_{2}+2 \sigma \gamma_{1}+b \gamma_{1}\right) x^{2} z+\left(\frac{\rho^{2}(b-1)^{2}}{4 \sigma^{2}}-r \gamma_{1} \gamma_{2}-2 \sigma \gamma_{4}\right) x^{2}+ \\
+\left(\frac{\rho^{2}(b-1)}{2 \sigma}+\gamma_{1} \gamma_{2}-2 r \sigma \gamma_{1}+2 r \gamma_{3}+2 \sigma \gamma_{4}+\sigma \gamma_{1} \gamma_{2}-\frac{\sigma}{b}\right) x y+ \\
+\left(2 \sigma \gamma_{1}-2 \gamma_{3}-\sigma \gamma_{1} \gamma_{2}+\frac{\rho^{2}}{4}\right) y^{2}+\left(\frac{\rho^{2}}{4}-2 b \gamma_{3}\right) z^{2}= \\
=A_{1} x^{4}+A_{2} x^{2} z+A_{3} z^{2}+B_{1} x^{2}+B_{2} x y+B_{3} y^{2}
\end{gathered}
$$

where

$$
\begin{gathered}
A_{1}=-\gamma_{1}, A_{2}=\gamma_{1}\left(\gamma_{2}+2 \sigma+b\right), A_{3}=\frac{\rho^{2}}{4}-2 b \gamma_{3}, \\
B_{1}=\frac{\left.\rho^{2}(b-1)^{2}\right)}{4 \sigma^{2}}-r \gamma_{1} \gamma_{2}-2 \sigma \gamma_{4}=C_{1}-2 \sigma \gamma_{4}, \\
B_{2}=\frac{\rho^{2}(b-1)}{2 \sigma}+\gamma_{1} \gamma_{2} 2 r \sigma \gamma_{1}+2 r \gamma_{3}+\sigma \gamma_{1} \gamma_{2}-\frac{\sigma}{b}+2 \sigma \gamma_{4}=C_{2}+2 \sigma \gamma_{4}, \\
B_{3}=2 \sigma \gamma_{1}-2 \gamma_{3}-\sigma \gamma_{1} \gamma_{2}+\frac{\rho^{2}}{4} .
\end{gathered}
$$

IV. a. Under the conditions $A_{1} \neq 0$ and $B_{3} \neq 0$ we transform the relation for $R$ :

$$
\left.R=A_{1}\right)\left(x^{2}+\frac{A_{2}}{2 A_{1}} z\right)^{2}+B_{3}\left(y+\frac{B_{2}}{2 B_{3}} x\right)^{2}+\frac{4 B_{1} B_{3}-B_{2}^{2}}{4 B_{3}} x^{2}+\frac{4 A_{1} A_{3}-A_{2}^{2}}{4 A_{1}} z^{2} .
$$

Then

$$
\left.\begin{array}{l}
A_{1}<0 \\
B_{3}<0 \\
4 A_{1} A_{3}-A_{2}^{2} \geq 0 \\
4 B_{1} B_{3}-B_{2}^{2} \geq 0
\end{array}\right\} \Rightarrow R \leq 0 \quad \forall x, y, z
$$

Now we analyze the signs of four addends in (30):

$$
A_{1}=-\gamma_{1}<0 \Leftrightarrow \gamma_{1}>0,
$$




$$
\begin{gathered}
B_{3}<0 \Leftrightarrow 2 \gamma_{3}>2 \sigma \gamma_{1}-\sigma \gamma_{1} \gamma_{2}+\frac{\rho^{2}}{4} \\
0 \leq 4 A_{1} A_{3}-A_{2}^{2}=-4 \gamma_{1}\left(\frac{\rho^{2}}{4}-2 b \gamma_{3}\right)-\gamma_{1}^{2}\left(\gamma_{2}+2 \sigma+b\right)^{2} .
\end{gathered}
$$

Since according to $(32) \gamma_{1}>0$, from (34) it follows that

$$
-\gamma_{1}\left(\gamma_{2}+2 \sigma+b\right)^{2}-\rho^{2}+8 b \gamma_{3} \geq 0 \Leftrightarrow 2 \gamma_{3} \geq \frac{\rho^{2}}{4 b}+\frac{\gamma_{1}}{4 b}\left(\gamma_{2}+2 \sigma+b\right)^{2} .
$$

Then

$$
\begin{aligned}
& 4 B_{1} B_{3}-B_{2}^{2}=4 B_{3}\left(C_{1}-2 \sigma \gamma_{4}\right)-\left(C_{2}+2 \sigma \gamma_{4}\right)^{2}=4 B_{3} C_{1}-8 \sigma B_{3} \gamma_{4}-C_{2}^{2}-4 \sigma C_{2} \gamma_{4}-4 \sigma^{2} \gamma_{4}^{2}= \\
& =-4 \sigma^{2} \gamma_{4}^{2}+4 \gamma_{4} \sigma\left(-2 B_{3}-C_{2}\right)+4 B_{3} C_{1}-C_{2}^{2} .
\end{aligned}
$$

The relation $4 B_{1} B_{3}-B_{2}^{2}$ is a quadratic polynomial in $\gamma_{4}$ with a negative coefficient of $\gamma_{4}^{2}$. Therefore for the inequality $4 B_{1} B_{3}-B_{2}^{2} \geq 0$ to be satisfied, it is necessary that the corresponding quadratic equation has a real root, i.e. a positive discriminant:

$$
\begin{gathered}
D_{\gamma_{4}}=16 \sigma^{2}\left(4 B_{3}^{2}+C_{2}^{2}+4 B_{3} C_{2}\right)+16 \sigma^{2}\left(4 B_{3} C_{1}-C_{2}^{2}\right)= \\
=16 C_{2}^{2} \sigma^{2}+64 \sigma^{2} B_{3}^{2}+64 \sigma^{2} B_{3} C_{2}+64 \sigma^{2} B_{3} C_{1}-16 \sigma^{2} C_{2}^{2}=64 \sigma^{2} B_{3}\left(B_{3}+C_{2}+C_{1}\right) .
\end{gathered}
$$

Since $B_{3}<0$ according to (31), we obtain $D_{\gamma_{4}} \geq 0 \Leftrightarrow B_{3}+C_{1}+C_{2} \leq 0$. Then

$$
\begin{gathered}
B_{3}+C_{1}+C_{2}=2 \sigma \gamma_{1}-2 \gamma_{3}-\sigma \gamma_{1} \gamma_{2}+\frac{\rho^{2}}{4}+\frac{\rho^{2}(b-1)^{2}}{4 \sigma^{2}}+ \\
-r \gamma_{1} \gamma_{2}+\frac{\rho^{2}(b-1)}{2 \sigma}+\gamma_{1} \gamma_{2}-2 r \sigma \gamma_{1}+2 r \gamma_{3}+\sigma \gamma_{1} \gamma_{2}-\frac{\sigma}{b}= \\
=\gamma_{3}(2 r-2)-\gamma_{1}(-2 \sigma+2 r \sigma)-\gamma_{1} \gamma_{2}(\sigma+r-\sigma-1)+\frac{\rho^{2}}{4}+\frac{\rho^{2}(b-1)^{2}}{4 \sigma^{2}}+ \\
+\frac{\rho^{2}(b-1)}{2 \sigma}-\frac{\sigma}{b}=2 \gamma_{3}(r-1)-2 \sigma \gamma_{1}(r-1)-\gamma_{1} \gamma_{2}(r-1)+\frac{\rho^{2}}{4}+ \\
+\frac{\rho^{2}(b-1)^{2}}{4 \sigma^{2}}+\frac{\rho^{2}(b-1)}{2 \sigma}-\frac{\sigma}{b} .
\end{gathered}
$$

Thus, for

$$
\left.2 \gamma_{3}(r)-1\right) \leq 2 \sigma \gamma_{1}(r-1)+\gamma_{1} \gamma_{2}(r-1)-\frac{\rho^{2}}{4}-\frac{\rho^{2}(b-1)^{2}}{4 \sigma^{2}}-\frac{\rho^{2}(b-1)}{2 \sigma}+\frac{\sigma}{b}
$$

there exists $\gamma_{4}$ such that $4 B_{1} B_{3}-B_{2}^{2} \geq 0$.

Note that in (36) a part of the right-hand side of the inequality can be transformed in the following way

$$
\frac{\rho^{2}}{4}+\frac{\rho^{2}(b-1)^{2}}{4 \sigma^{2}}+\frac{\rho^{2}(b-1)}{2 \sigma}=\frac{\rho^{2}}{\sigma^{2}}\left(\frac{\sigma^{2}}{4}+\frac{(b-1)^{2}}{4}+\frac{\sigma(b-1)}{2}\right)=\frac{\rho^{2}(\sigma+b-1)^{2}}{4 \sigma^{2}} .
$$


Taking into account (32), (33), (35), (36), (37), conditions (31) become

$$
\left.\begin{array}{l}
\gamma_{1}>0 \\
2 \gamma_{3}>2 \sigma \gamma_{1}-\sigma \gamma_{1} \gamma_{2}+\frac{\rho^{2}}{4} \\
2 \gamma_{3} \geq \frac{\rho^{2}}{4 b}+\frac{\gamma_{1}}{4 b}\left(\gamma_{2}+2 \sigma+b\right)^{2} \\
2(r-1) \gamma_{3} \leq 2 \sigma \gamma_{1}(r-1)+\gamma_{1} \gamma_{2}(r-1)-\frac{\rho^{2}(b+\sigma-1)^{2}}{4 \sigma^{2}}+\frac{\sigma}{b}
\end{array}\right\} \Rightarrow R \leq 0 \quad \forall x, y, z .
$$

For obtaining (30) it is assumed that $A_{1} \neq 0, B_{3} \neq 0$. Let us analyze the cases $A_{1}=0$ and $B_{3}=0$.

IV. b. Consider $B_{3}=0$. In this case we have

$$
R=A_{1} x^{4}+A_{2} x^{2} z+A_{3} z^{2}+B_{1} x^{2}+B_{2} x y
$$

i.e. it is impossible to choose $\gamma_{1}, \gamma_{2}, \gamma_{3}, \gamma_{4}$ in such a way that $R \leq 0$ is valid $\forall x, y, z$.

IV. c. Consider the case $\gamma_{1}=A_{1}=0$ and $B_{3} \neq 0$. Then

$$
A_{2}=\gamma_{1}\left(\gamma_{2}+2 \sigma+b\right)=0
$$

and

$$
R=A_{3} z^{2}+B_{1} x^{2}+B_{2} x y+B_{3} y^{2}=A_{3} z^{2}+B_{3}\left(y+\frac{B_{2}}{2 B_{3}} x\right)^{2}+\frac{4 B_{1} B_{3}-B_{2}^{2}}{4 B_{3}} x^{2} .
$$

In this case

$$
\left.\begin{array}{l}
A_{3} \leq 0 \\
B_{3}<0 \\
4 B_{1} B_{3}-B_{2}^{2} \geq 0
\end{array}\right\} \Rightarrow R \leq 0 \quad \forall x, y, z .
$$

The second and third conditions are similar to the second and fourth conditions in (31). Consequently it remains to consider

$$
0 \geq A_{3}=\frac{\rho^{2}}{4}-2 b \gamma_{3} \Leftrightarrow 2 \gamma_{3} \geq \frac{\rho^{2}}{4 b}
$$

The latter inequality, obtained under the assumption $A_{1}=0$, coincides with condition (33) if in (33) we take $\gamma_{1}=0$. Thus, the conditions on the running parameters $\gamma_{1}, \gamma_{2}, \gamma_{3}, \gamma_{4}$, obtained under the condition $A_{1}=0, B_{3} \neq 0$, can be joined with those, obtained under the condition $A_{1} \neq 0, B_{3} \neq 0$, i.e.

$$
\left.\begin{array}{l}
\gamma_{1} \geq 0 \\
2 \gamma_{3}>2 \sigma \gamma_{1}-\sigma \gamma_{1} \gamma_{2}+\frac{\rho^{2}}{4} \\
2 \gamma_{3} \geq \frac{\rho^{2}}{4 b}+\frac{\gamma_{1}}{4 b}\left(\gamma_{2}+2 \sigma+b\right)^{2} \\
2(r-1) \gamma_{3} \leq 2 \sigma \gamma_{1}(r-1)+\gamma_{1} \gamma_{2}(r-1)-\frac{\rho^{2}(b+\sigma-1)^{2}}{4 \sigma^{2}}+\frac{\sigma}{b}
\end{array}\right\}
$$


V. For $r-1>0$ conditions (40) can be transformed in the following way:

$$
\left\{\begin{array}{l}
\gamma_{1} \geq 0 \\
2 \gamma_{3}>\frac{\rho^{2}}{4}+2 \sigma \gamma_{1}-\sigma \gamma_{1} \gamma_{2}, \\
2 \gamma_{3} \geq \frac{\rho^{2}}{4 b}+\frac{\gamma_{1}}{4 b}\left(\gamma_{2}+2 \sigma+b\right)^{2} \\
2 \gamma_{3} \leq 2 \sigma \gamma_{1}+\gamma_{1} \gamma_{2}-\frac{\rho^{2}(b+\sigma-1)^{2}}{4 \sigma^{2}(r-1)}+\frac{\sigma}{b(r-1)}
\end{array}\right.
$$

For the existence of $\gamma_{3}$ it is necessary and sufficient that

$$
\left\{\begin{array}{l}
\frac{\rho^{2}}{4}+\gamma_{1}\left(2 \sigma-\sigma \gamma_{2}\right)<\gamma_{1}\left(2 \sigma+\gamma_{2}\right)-\frac{\rho^{2}(b+\sigma-1)^{2}}{4 \sigma^{2}(r-1)}+\frac{\sigma}{b(r-1)} \\
\frac{\rho^{2}}{4 b}+\frac{\gamma_{1}}{4 b}\left(2 \sigma+b+\gamma_{2}\right)^{2} \leq \gamma_{1}\left(2 \sigma+\gamma_{2}\right)-\frac{\rho^{2}(b+\sigma-1)^{2}}{4 \sigma^{2}(r-1)}+\sigma(r-1)
\end{array}\right.
$$

We now analyze the above inequalities.

V. a. Consider the first inequality from (42) for different $\gamma_{2}$

Let $\gamma_{2}=0$. Then the inequality is equivalent to

$$
\frac{\rho^{2}}{4}+\frac{\rho^{2}(b+\sigma-1)^{2}}{4 \sigma^{2}(r-1)}-\frac{\langle\sigma}{b(r-1)}<0 .
$$

If $\gamma_{2}>0$, then we obtain the condition

$$
\gamma_{1}>\frac{1}{\gamma_{2}(\sigma+1)}\left(\frac{\rho^{2}}{4}+\frac{\rho^{2}(b+\sigma-1)^{2}}{4 \sigma^{2}(r-1)}-\frac{\sigma}{b(r-1)}\right),
$$

and if $\gamma_{2}<0$, then

$$
\gamma_{1}<\frac{1}{\gamma_{2}(\sigma+1)}\left(\frac{\rho^{2}}{4}+\frac{\rho^{2}(b+\sigma-1)^{2}}{4 \sigma^{2}(r-1)}-\frac{\sigma}{b(r-1)}\right) .
$$

In the latter case it is required that

$$
\frac{\rho^{2}}{4}+\frac{\rho^{2}(b+\sigma-1)^{2}}{4 \sigma^{2}(r-1)}-\frac{\sigma}{b(r-1)}<0
$$

since, according to (40), we have $\gamma_{1} \geq 0$. Thus, since the cases of $\gamma_{2}=0$ and $\gamma_{2}<0$ impose the same condition on the parameters of the system, they can be joined.

V. b. From the second inequality of system (42) we obtain the condition for $\gamma_{1}$ :

$$
\frac{\left(2 \sigma-b+\gamma_{2}\right)^{2}}{4 b} \gamma_{1} \leq-\left(\frac{\rho^{2}}{4 b}+\frac{\rho^{2}(b+\sigma-1)^{2}}{4 \sigma^{2}(r-1)}-\frac{\sigma}{b(r-1)}\right) \text {. }
$$

If $\gamma_{2}=b-2 \sigma$, then it is required that

$$
\frac{\rho^{2}}{4 b}+\frac{\rho^{2}(b+\sigma-1)^{2}}{4 \sigma^{2}(r-1)}-\frac{\sigma}{b(r-1)} \leq 0 .
$$

If $\gamma_{2} \neq b-2 \sigma$, then we obtain the following condition for $\gamma_{1}$

$$
\gamma_{1} \leq-\frac{4 b}{\left(2 \sigma-b+\gamma_{2}\right)^{2}}\left(\frac{\rho^{2}}{4 b}+\frac{\rho^{2}(b+\sigma-1)^{2}}{4 \sigma^{2}(r-1)}-\frac{\sigma}{b(r-1)}\right) .
$$


According to (40), $\gamma_{1} \geq 0$ is valid. This implies the following

$$
\frac{\rho^{2}}{4 b}+\frac{\rho^{2}(b+\sigma-1)^{2}}{4 \sigma^{2}(r-1)}-\frac{\sigma}{b(r-1)} \leq 0
$$

Thus, inequality (44) holds true also for $\gamma_{2}=b-2 \sigma$ and $\gamma_{2} \neq b-2 \sigma$.

Condition (44) must be satisfied for any sign of $\gamma_{2}$. If, in addition, condition (43) is also satisfied, then in function (27) we can take $\gamma_{2} \leq 0$ and in this case there exist $\gamma_{1}, \gamma_{3}, \gamma_{4}$ such that the relation

$$
R=-\sigma z+\frac{\rho^{2} z^{2}}{4}+\frac{\rho^{2}}{4}\left(y+\frac{b-1}{\sigma} x\right)^{2}+\dot{\theta} \leq 0 \quad \forall x, y, z
$$

is valid. If inequality (43) is not satisfied, then in (27) it must be taken $\gamma_{2}>0$ and, except for condition (44), it is necessary to find conditions for the existence of $\gamma_{1}$.

In this case we obtain the following family

$$
\left[\begin{array}{l}
\left\{\begin{array}{l}
\frac{\rho^{2}}{4 b}+\frac{\rho^{2}(b+\sigma-1)^{2}}{4 \sigma^{2}(r-1)}-\frac{\sigma}{b(r-1)} \leq 0 \\
\frac{\rho^{2}}{4}+\frac{\rho^{2}(b+\sigma-1)^{2}}{4 \sigma^{2}(r-1)}-\frac{\sigma}{b(r-1)}<0
\end{array}\right. \\
\left\{\begin{array}{l}
\frac{\rho^{2}}{4 b}+\frac{\rho^{2}(b+\sigma-1)^{2}}{4 \sigma^{2}(r-1)}-\frac{\sigma}{b(r-1)} \leq 0 \\
\gamma_{2}>0, \gamma_{2} \neq b-2 \sigma, \\
\frac{1}{\gamma_{2}(\sigma+1)}\left(\frac{\rho^{2}}{4}+\frac{\rho^{2}(b+\sigma-1)^{2}}{4 \sigma^{2}(r-1)}-\frac{\sigma}{b(r-1)}\right)< \\
<-\frac{4 b}{\left(2 \sigma-b+\gamma_{2}\right)^{2}}\left(\frac{\rho^{2}}{4 b}+\frac{\rho^{2}(b+\sigma-1)^{2}}{4 \sigma^{2}(r-1)}-\frac{\sigma}{b(r-1)}\right)
\end{array}\right) .
\end{array}\right.
$$

V. c.

Consider the first condition of (45)

$$
\left\{\begin{array}{l}
\frac{\rho^{2}}{4 b}+\frac{\rho^{2}(b+\sigma-1)^{2}}{4 \sigma^{2}(r-1)}-\frac{\sigma}{b(r-1)} \leq 0 \\
\frac{\rho^{2}}{4}+\frac{\rho^{2}(b+\sigma-1)^{2}}{4 \sigma^{2}(r-1)}-\frac{\sigma}{b(r-1)}<0 .
\end{array}\right.
$$

Substituting the value $\rho$ from (22), we obtain

$$
\begin{gathered}
\left\{\begin{array}{l}
\frac{\sigma^{2}}{4 b\left((r-1) \sigma-b^{2}+b+\sigma b\right)}+\frac{(b+\sigma-1)^{2}}{4(r-1)\left((r-1) \sigma-b^{2}+b+\sigma b\right)}-\frac{\sigma}{b(r-1)} \leq 0 \\
\frac{\sigma^{2}}{4\left((r-1) \sigma-b^{2}+b+\sigma b\right)}+\frac{(b+\sigma-1)^{2}}{4(r-1)\left((r-1) \sigma-b^{2}+b+\sigma b\right)}-\frac{\sigma}{b(r-1)}<0 .
\end{array}\right. \\
\left\{\begin{array}{l}
r-1 \geq \frac{b(b+\sigma-1)^{2}-4 \sigma\left(b+\sigma b-b^{2}\right)}{3 \sigma^{2}} \\
\sigma^{2}(r-1)(b-4)<4 \sigma\left(\sigma b+b-b^{2}\right)-b(b+\sigma-1)^{2}
\end{array}\right.
\end{gathered}
$$


V. d. Consider the second condition of (45):

$$
\left\{\begin{array}{l}
\frac{\rho^{2}}{4 b}+\frac{\rho^{2}(b+\sigma-1)^{2}}{4 \sigma^{2}(r-1)}-\frac{\sigma}{b(r-1)} \leq 0, \\
\gamma_{2}>0, \gamma_{2} \neq b-2 \sigma, \\
\frac{1}{\gamma_{2}(\sigma+1)}\left(\frac{\rho^{2}}{4}+\frac{\rho^{2}(b+\sigma-1)^{2}}{4 \sigma^{2}(r-1)}-\frac{\sigma}{b(r-1)}\right)< \\
<-\frac{4 b}{\left(2 \sigma-b+\gamma_{2}\right)^{2}}\left(\frac{\rho^{2}}{4 b}+\frac{\rho^{2}(b+\sigma-1)^{2}}{4 \sigma^{2}(r-1)}-\frac{\sigma}{b(r-1)}\right) .
\end{array}\right.
$$

From Item V. c. it is known that the first inequality of system is equivalent to the relation

$$
r-1 \geq \frac{b(b+\sigma-1)^{2}-4 \sigma\left(b+\sigma b-b^{2}\right)}{3 \sigma^{2}} .
$$

Next we transform the following inequality

$$
\begin{gathered}
\frac{1}{\gamma_{2}(\sigma+1)}\left(\frac{\rho^{2}}{4}+\frac{\rho^{2}(b+\sigma-1)^{2}}{4 \sigma^{2}(r-1)}-\frac{\sigma}{b(r-1)}\right)<-\frac{4 b}{\left(2 \sigma-b+\gamma_{2}\right)^{2}}\left(\frac{\rho^{2}}{4 b}+\frac{\rho^{2}(b+\sigma-1)^{2}}{4 \sigma^{2}(r-1)}-\frac{\sigma}{b(r-1)}\right) \\
\Leftrightarrow \\
\left(2 \sigma-b+\gamma_{2}\right)^{2}\left(\frac{\rho^{2}}{4}+\frac{\rho^{2}(b+\sigma-1)^{2}}{4 \sigma^{2}(r-1)}-\frac{\sigma}{b(r-1)}\right)+4 b \gamma_{2}(\sigma+1)\left(\frac{\rho^{2}}{4 b}+\frac{\rho^{2}(b+\sigma-1)^{2}}{4 \sigma^{2}(r-1)}-\frac{\sigma}{b(r-1)}\right)<0 \\
\Leftrightarrow \\
\left(2 \sigma-b+\gamma_{2}\right)^{2}\left[b(b+\sigma-1)^{2}-4 \sigma\left(\sigma b+b-b^{2}\right)+\sigma^{2}(r-1)(b-4)\right]+ \\
+4 b \gamma_{2}(\sigma+1)\left(b(b+\sigma-1)^{2}>4 \sigma\left(\sigma b+b-b^{2}\right)-3 \sigma^{2}(r-1)\right)<0 .
\end{gathered}
$$

The left-hand side of the inequality is a quadratic polynomial in $\gamma_{2}$. If the coefficient of $\gamma_{2}^{2}$ is negative, i.e.

$$
b(b+\sigma-1)^{2}-4 \sigma\left(\sigma b+b-b^{2}\right)+\sigma^{2}(r-1)(b-4)<0,
$$

then there exists $\gamma_{2}>0$ satisfying inequality (47).

If the coefficient is equal to 0 , i.e.

$$
b(b+\sigma-1)^{2}-4 \sigma\left(\sigma b+b-b^{2}\right)+\sigma^{2}(r-1)(b-4)=0,
$$

then the relation

$$
4 b \gamma_{2}(\sigma+1)\left[b(b+\sigma-1)^{2}-4 \sigma\left(\sigma b+b-b^{2}\right)-3 \sigma^{2}(r-1)\right]<0
$$

has to be valid. By (46), $\gamma_{2}>0$ is satisfied and $b(b+\sigma-1)^{2}-4 \sigma\left(b+\sigma b-b^{2}\right)-3 \sigma^{2}(r-1) \leq 0$, Then the inequality

$$
4 b \gamma_{2}(\sigma+1)\left[b(b+\sigma-1)^{2}-4 \sigma\left(\sigma b+b-b^{2}\right)-3 \sigma^{2}(r-1)\right]<0
$$

is valid.

If the coefficient is positive, i.e.

$$
b(b+\sigma-1)^{2}-4 \sigma\left(\sigma b+b-b^{2}\right)+\sigma^{2}(r-1)(b-4)>0,
$$


then for the existence of $\gamma_{2}>0$, satisfying (47), it is necessary and sufficient that there exist two different real roots of the equation

$$
\begin{array}{r}
\left(2 \sigma-b+\gamma_{2}\right)^{2}\left[b(b+\sigma-1)^{2}-4 \sigma\left(\sigma b+b-b^{2}\right)+\sigma^{2}(r-1)(b-4)\right]+ \\
+4 b \gamma_{2}(\sigma+1)\left(b(b+\sigma-1)^{2}-4 \sigma\left(\sigma b+b-b^{2}\right)-3 \sigma^{2}(r-1)\right)=0
\end{array}
$$

and the largest root must be positive. This condition corresponds to the second condition of the theorem.

V. e. Relations (11)-(14) imply the inequality

$$
R=-\sigma z+\frac{\rho^{2} z^{2}}{4}+\frac{\rho^{2}}{4}\left(y+\frac{b-1}{\sigma} x\right)^{2}+\dot{\theta} \leq 0 \quad \forall x, y, z .
$$

Consequently, (29) is valid:

$$
2\left(\lambda_{1}+\lambda_{2}+s \lambda_{3}\right)+2 \dot{V} \leq-(\sigma+2 b+1)-s(\sigma+1)+(1-s)\left[(\sigma-1)^{2}+4 \sigma r\right]^{\frac{1}{2}} .
$$

VI. To apply Theorem 2, we consider inequality (29) for $s=0$ :

$$
2\left(\lambda_{1}+\lambda_{2}+\dot{V}\right) \leq-(\sigma+2 b+1)+\left[(\sigma-1)^{2}+4 \sigma r\right]^{\frac{1}{2}} .
$$

Then we find conditions for the parameters of system $\sigma, b, r$ for which $2\left(\lambda_{1}+\lambda_{2}+\dot{V}\right)<0$. We have

$$
-(\sigma+2 b+1)+\left[(\sigma-1)^{2}+4 \sigma r\right]^{\frac{1}{2}}<0 \Leftrightarrow\left[(\sigma-1)^{2}+4 \sigma r\right]^{\frac{1}{2}}<(\sigma+2 b+1) .
$$

The parameters of system $\sigma, b$ are positive numbers, i.e. $\sigma+2 b+1>0$. Therefore after squaring two sides of inequality (49) we obtain

$$
\begin{gathered}
\sigma^{2}-2 \sigma+1+4 \sigma r<\sigma^{2}+1+4 b^{2}+2 \sigma+4 \sigma b+4 b \\
\Leftrightarrow \\
4 \sigma r<4 b^{2}+4 \sigma+4 \sigma b+4 b \Leftrightarrow \sigma r<(b+1)(b+\sigma) .
\end{gathered}
$$

Thus, taking into account (21), we obtain condition (16) for which

$$
2\left(\lambda_{1}+\lambda_{2}+\dot{V}\right)<0 .
$$

Then Theorem 2 can be applied, i.e. any bounded on $[0 ;+\infty)$ solution of system (1) tends to a certain equilibrium as $t \rightarrow+\infty$.

VII. Inequality (15) is obtained in [39].

VIII. To apply Theorem 1 , we take $s \neq 0$ and (29):

$$
\begin{gathered}
2\left(\lambda_{1}+\lambda_{2}+s \lambda_{3}+\dot{V}\right) \leq-(\sigma+2 b+1)-s(\sigma+1)+(1-s)\left[(\sigma-1)^{2}+4 \sigma r\right]^{\frac{1}{2}}= \\
=-s\left(\sigma+1+\left[(\sigma-1)^{2}+4 \sigma r\right]^{\frac{1}{2}}\right)-(\sigma+2 b+1)+\left[(\sigma-1)^{2}+4 \sigma r\right]^{\frac{1}{2}} .
\end{gathered}
$$

Find $s$ such that the relation $2\left(\lambda_{1}+\lambda_{2}+s \lambda_{3}+\dot{V}\right)<0$ is valid:

$$
\begin{gathered}
-s\left(\sigma+1+\left[(\sigma-1)^{2}+4 \sigma r\right]^{\frac{1}{2}}\right)-(\sigma+2 b+1)+\left[(\sigma-1)^{2}+4 \sigma r\right]^{\frac{1}{2}}<0 \\
\Leftrightarrow \\
-(\sigma+2 b+1)+\left[(\sigma-1)^{2}+4 \sigma r\right]^{\frac{1}{2}}<s\left(\sigma+1+\left[(\sigma-1)^{2}+4 \sigma r\right]^{\frac{1}{2}}\right) .
\end{gathered}
$$


The parameter $\sigma$ of system (1) is a positive number. Therefore the coefficient of $s, \sigma+1+[(\sigma-$ $\left.1)^{2}+4 \sigma r\right]^{\frac{1}{2}}$, is greater than 0 . In this case if inequality (50) is divided by $\sigma+1+\left[(\sigma-1)^{2}+4 \sigma r\right]^{\frac{1}{2}}$, then the sign is not changed, i.e. we have

$$
s>s_{0}=\frac{-(\sigma+2 b+1)+\left[(\sigma-1)^{2}+4 \sigma r\right]^{\frac{1}{2}}}{\sigma+1+\left[(\sigma-1)^{2}+4 \sigma r\right]^{\frac{1}{2}}} .
$$

Thus, in the case when (11)-(14) are satisfied and $s$ satisfies (51) we have $2\left(\lambda_{1}+\lambda_{2}+s \lambda_{3}+\dot{V}\right)<0$.

Under the hypothesis of Theorem 1 we have $s \in[0,1)$. However according to (51) the relation $s>s_{0}$ must be valid. The case $s=0$ is already considered. Obviously, $\sigma+1+\left[(\sigma-1)^{2}+4 \sigma r\right]^{\frac{1}{2}}>0$. Therefore for $s_{0}>0$ to be valid, it is required that

$$
-(\sigma+2 b+1)+\left[(\sigma-1)^{2}+4 \sigma r\right]^{\frac{1}{2}}>0 \Leftrightarrow\left[(\sigma-1)^{2}+4 \sigma r\right]^{\frac{1}{2}}>\sigma+2 b+1 .
$$

The relation $\sigma+2 b+1 \geq 0$ is always satisfied since the parameters $\sigma, b$ of system $(1)$ are positive numbers. Thus, after squaring inequality (52) we obtain

$$
\sigma^{2}-2 \sigma+1+4 \sigma r>\sigma^{2}+1+4 b^{2}+2 \sigma+4 \sigma b+4 b \Leftrightarrow \sigma r>(b+1)(b+\sigma) .
$$

Next we find a condition for which $s_{0}<1$ :

$$
\begin{gathered}
\frac{-(\sigma+2 b+1)+\left[(\sigma-1)^{2}+4 \sigma r\right]^{\frac{1}{2}}}{\sigma+1+\left[(\sigma-1)^{2}+4 \sigma r\right]^{\frac{1}{2}}}<1 \\
\Leftrightarrow \\
-(\sigma+2 b+1)+\left[(\sigma-1)^{2}+4 \sigma r\right]^{\frac{1}{2}}<\sigma+1+\left[(\sigma-1)^{2}+4 \sigma r\right]^{\frac{1}{2}} \\
\Leftrightarrow \Leftrightarrow+1+b
\end{gathered}
$$

The latter inequality is always valid. The parameters of system (1) are positive numbers and therefore

$$
(b+1)(b+\sigma)>(b-\sigma)(b-1)
$$

is always satisfied, i.e. from conditions (21), (53) it remains only condition (53). Thus, it is obtained condition (17) under which the relation $2\left(\lambda_{1}+\lambda_{2}+s \lambda_{3}+\dot{V}\right)<0$ is satisfied and Theorem 1 can be applied. Consequently, $\operatorname{dim}_{\mathrm{L}} K \leq 2+s$ for $s$, satisfying (51) and (17). Thus,

$$
\operatorname{dim}_{\mathrm{L}} K \leq 3-\frac{2(\sigma+b+1)}{\sigma+1+\sqrt{(\sigma-1)^{2}+4 \sigma r}}
$$

\subsection{Proof of Corollary 3}

I. We consider inequalities (11), (12), which are sufficient conditions for the theorem to be valid:

$$
\left\{\begin{array}{l}
r-1 \geq \frac{b(b+\sigma-1)^{2}-4 \sigma\left(b+\sigma b-b^{2}\right)}{3 \sigma^{2}}, \\
\sigma^{2}(r-1)(b-4) \leq 4 \sigma\left(\sigma b+b-b^{2}\right)-b(b+\sigma-1)^{2} .
\end{array}\right.
$$

Since it is considered $1<b \leq 2, \sigma>0$, we get

$$
\left\{\begin{array}{l}
r-1 \geq \frac{b(b+\sigma-1)^{2}-4 \sigma\left(b+\sigma b-b^{2}\right)}{3 \sigma^{2}}, \\
r-1 \leq \frac{b(b+\sigma-1)^{2}-4 \sigma\left(\sigma b+b-b^{2}\right)}{\sigma^{2}(4-b)} .
\end{array}\right.
$$


Consider the right-hand side of these inequalities. Both denominators are positive and the numerators are the same. The numerators can be transformed as

$$
b(b+\sigma-1)^{2}-4 \sigma\left(b+\sigma b-b^{2}\right)=-3 \sigma^{2} b+\left(-6 b+6 b^{2}\right) \sigma+b(b-1)^{2} .
$$

Since the discriminant of the corresponding quadratic equation $D_{\sigma}=48 b^{2}(b-1)^{2}$ is positive, we have the following real roots

$$
\sigma_{1,2}=\left(1 \mp \frac{2 \sqrt{3}}{3}\right)(b-1)
$$

Therefore, since coefficient of $\sigma^{2}$ in (55) is negative, the right-hand sides of inequalities (54) are positive if

$$
\sigma \in\left(\sigma_{1}, \sigma_{2}\right)
$$

and are negative if

$$
\sigma \in\left(-\infty, \sigma_{1}\right) \cup\left(\sigma_{2},+\infty\right)
$$

If $\sigma \in\left(\sigma_{2},+\infty\right)$, then

$$
\max \left[0, \frac{b(b+\sigma-1)^{2}-4 \sigma\left(b+\sigma b-b^{2}\right)}{3 \sigma^{2}}, \frac{b(b+\sigma-1)^{2}-4 \sigma\left(\sigma b+b-b^{2}\right)}{\sigma^{2}(4-b)}\right]=0,
$$

and three inequalities (10), (11), and (12) are equivalent to $r-1)>0$.

If $\sigma \in\left(0, \sigma_{2}\right)$, then

$$
\begin{gathered}
\max \left[0, \frac{b(b+\sigma-1)^{2}-4 \sigma\left(b+\sigma b-b^{2}\right)}{3 \sigma^{2}}, \frac{b(b+\sigma-1)^{2}-4 \sigma\left(\sigma b+b-b^{2}\right)}{\sigma^{2}(4-b)}\right]= \\
=\frac{b(b+\sigma-1)^{2}-4 \sigma\left(\sigma b+b-b^{2}\right)}{\sigma^{2}(4-b)},
\end{gathered}
$$

and three inequalities (10), (11), and (12) are equivalent to the following inequality

$$
r=1>\frac{b(b+\sigma-1)^{2}-4 \sigma\left(\sigma b+b-b^{2}\right)}{\sigma^{2}(4-b)} \text {. }
$$

II. Consider (16):

$$
(b-\sigma)(b-1)<\sigma r<(b+1)(b+\sigma),
$$

and find conditions on parameters $\sigma, b, r$ such that

$$
(b-\sigma)(b-1)<\sigma r .
$$

Since $\sigma>0$, inequality (57) is equivalent to the following one

$$
\frac{b(b-1-\sigma)}{\sigma}<r-1
$$

Since $1<b \leq 2$, the left-hand side of inequality (58) is negative if $\sigma>1$ and positive if $\sigma<1$.

III. Thus, if $\sigma \in\left(\max \left(\sigma_{2}, 1\right),+\infty\right)$, then

$$
\max \left[0, \frac{b(b+\sigma-1)^{2}-4 \sigma\left(b+\sigma b-b^{2}\right)}{3 \sigma^{2}}, \frac{b(b+\sigma-1)^{2}-4 \sigma\left(\sigma b+b-b^{2}\right)}{\sigma^{2}(4-b)}, \frac{b(b-1-\sigma)}{\sigma}\right]=0 .
$$

Therefore, for Theorem 3 to be valid, only the inequality $r-1>0$ must be hold. 
IV. If $\sigma \in\left(0, \max \left(\sigma_{2}, 1\right)\right), 1<b \leq 2$, then

$$
\begin{gathered}
\frac{b(b-1-\sigma)}{\sigma} \leq \frac{b(b+\sigma-1)^{2}-4 \sigma\left(\sigma b+b-b^{2}\right)}{\sigma^{2}(4-b)} \\
\Leftrightarrow \Leftrightarrow(1+\sigma) b^{3}+\left(-\sigma^{2}+\sigma-2\right) b^{2}+(\sigma-1)^{2} b .
\end{gathered}
$$

Here the right-hand side is the third-order polynomial in $b$ and the corresponding roots are the following

$$
b_{1}=0, \quad b_{2}=\frac{(\sigma-1)^{2}}{\sigma+1}, \quad b_{3}=1 .
$$

The maximum of $\frac{(\sigma-1)^{2}}{\sigma+1}$ on the interval $\sigma \in\left(0, \max \left(\sigma_{2}, 1\right)\right)$ is 1 and the minimum is 0 . So $b_{1} \leq$ $b_{2} \leq b_{3}$. Thus, inequality (59) is valid on interval $1<b \leq 2$, since coefficient of $b^{3}$ in the right-hand side is positive. Then

$$
\begin{gathered}
\max \left[0, \frac{b(b+\sigma-1)^{2}-4 \sigma\left(b+\sigma b-b^{2}\right)}{3 \sigma^{2}}, \frac{b(b+\sigma-1)^{2}-4 \sigma\left(\sigma b+b-b^{2}\right)}{\sigma^{2}(4-b)}, \frac{b(b-1-\sigma)}{\sigma}\right]= \\
=\frac{b(b+\sigma-1)^{2}-4 \sigma\left(\sigma b+b-b^{2}\right)}{\sigma^{2}(4-b)}
\end{gathered}
$$

and if

$$
r-1>\frac{b(b+\sigma-1)^{2}-4 \sigma\left(\sigma b+b-b^{2}\right)}{\sigma^{2}(4-b)}
$$

Theorem 3 is valid.

V. Now consider condition (15) of Theorem 3 to obtain the remaining interval

$$
\sigma \in\left(0, \max \left(\sigma_{2}, 1\right)\right), \quad 0<r-1<\frac{b(b+\sigma-1)^{2}-4 \sigma\left(\sigma b+b-b^{2}\right)}{\sigma^{2}(4-b)} .
$$

If $\sigma>1$, then $\lambda_{0}=1$ and

$$
2 \sigma-b<(\sigma+1)\left(\sigma r^{-1}+1\right) \max _{\lambda \in\left[0, \lambda_{0}\right]} \frac{\lambda(b-\lambda)}{(\sqrt{r \sigma}-\sqrt{(\sigma-\lambda)(1-\lambda)})^{2}} .
$$

In this case, we obtain the following sufficient condition (for $\lambda=1$ in the right-hand side of inequality):

$$
2 \sigma-b<\frac{(\sigma+1)(\sigma+r)(b-1)}{r^{2} \sigma}
$$

which is transformed to the following quadratic inequality with respect to $r$

$$
\left(2 \sigma^{2}-\sigma b\right) r^{2}+(\sigma-\sigma b-b+1) r+\sigma^{2}-\sigma^{2} b-\sigma b+\sigma<0 .
$$

It is easy to check that the roots of the corresponding quadratic equation

$$
\begin{aligned}
& r_{1}=\frac{\sigma b-\sigma+b-1-\sqrt{(8 b-8) \sigma^{4}+\left(-4 b^{2}+12 b-8\right) \sigma^{3}+\left(-3 b^{2}+2 b+1\right) \sigma^{2}+2(b-1)^{2} \sigma+(b-1)^{2}}}{2 \sigma(2 \sigma-b)}, \\
& r_{2}=\frac{\sigma b-\sigma+b-1+\sqrt{(8 b-8) \sigma^{4}+\left(-4 b^{2}+12 b-8\right) \sigma^{3}+\left(-3 b^{2}+2 b+1\right) \sigma^{2}+2(b-1)^{2} \sigma+(b-1)^{2}}}{2 \sigma(2 \sigma-b)}
\end{aligned}
$$


exist and $r_{1}<0<r_{2}$ if $\sigma>1$ and $1<b \leq 2$. Since the coefficient of $r^{2}$ in (60) is positive provided that $\sigma$ and $b$ are from the considered domain, then we need to consider the relation $0<r-1<r_{1}-1$ only. If the inequality

$$
\begin{gathered}
\frac{b(b+\sigma-1)^{2}-4 \sigma\left(\sigma b+b-b^{2}\right)}{\sigma^{2}(4-b)}< \\
<\frac{\sigma b-\sigma+b-1+\sqrt{(8 b-8) \sigma^{4}+\left(-4 b^{2}+12 b-8\right) \sigma^{3}+\left(-3 b^{2}+2 b+1\right) \sigma^{2}+2(b-1)^{2} \sigma+(b-1)^{2}}}{2 \sigma(2 \sigma-b)}-1
\end{gathered}
$$

is valid, then Theorem 3 is hold for $\sigma \in\left(1, \max \left(\sigma_{2}, 1\right)\right), b \in(1,2], r-1>0$.

Since we consider $\sigma \in\left(1, \max \left(\sigma_{2}, 1\right)\right), b \in(1,2]$, then $\sigma^{2}(4-b)(2 \sigma-b)>0$ and inequality (61) is transformed to

$$
\begin{aligned}
& (-16 b+16) \sigma^{4}+\left(33 b^{2}-37 b+4\right) \sigma^{3}+\left(-8 b^{3}+5 b^{2}-b+4\right) \sigma^{2}+\left(-2 b^{4}-4 b^{3}-2 b^{2}\right) \sigma< \\
< & \sigma^{2}(4-b) \sqrt{(8 b-8) \sigma^{4}+\left(-4 b^{2}+12 b-8\right) \sigma^{3}+\left(-3 b^{2}+2 b+1\right) \sigma^{2}+2(b-1)^{2} \sigma+(b-1)^{2}} .
\end{aligned}
$$

This inequality is valid in the case of negative left-hand side, because $1<b \leq 2$. If the left-hand side is positive, we square both sides of the inequality:

$$
\begin{gathered}
\left((-16 b+16) \sigma^{4}+\left(33 b^{2}-37 b+4\right) \sigma^{3}+\left(-8 b^{3}+5 b^{2}-b+4\right) \sigma^{2}+\left(-2 b^{4}+4 b^{3}-2 b^{2}\right) \sigma\right)^{2}< \\
<\sigma^{4}(4-b)^{2}\left((8 b-8) \sigma^{4}+\left(-4 b^{2}+12 b-8\right) \sigma^{3}+\left(-3 b^{2}+2 b+1\right) \sigma^{2}+2(b-1)^{2} \sigma+(b-1)^{2}\right) \\
\Leftrightarrow \\
+\left(-1348 b^{4}+2884 b^{3}-1888 b^{2}+480 b-128\right) \sigma^{6}+\left(464 b^{5}-728 b^{4}+288 b^{3}-248 b^{2}+224 b\right) \sigma^{5}+ \\
+\left(68 b^{6}-332 b^{5}+404 b^{4}-116 b^{3}+8 b^{2}-32 b\right) \sigma^{4}+\left(-32 b^{7}+84 b^{6}-76 b^{5}+44 b^{4}-36 b^{3}+16 b^{2}\right) \sigma^{3}+ \\
+\left(-4 b^{8}+16 b^{7}-24 b^{6}+16 b^{5}-4 b^{4}\right) \sigma^{2}=: h_{1}(\sigma, b)=h_{1} .
\end{gathered}
$$

As is shown in Fig. 5, inequality (62) holds, and, therefore, inequality (61) is satisfied.
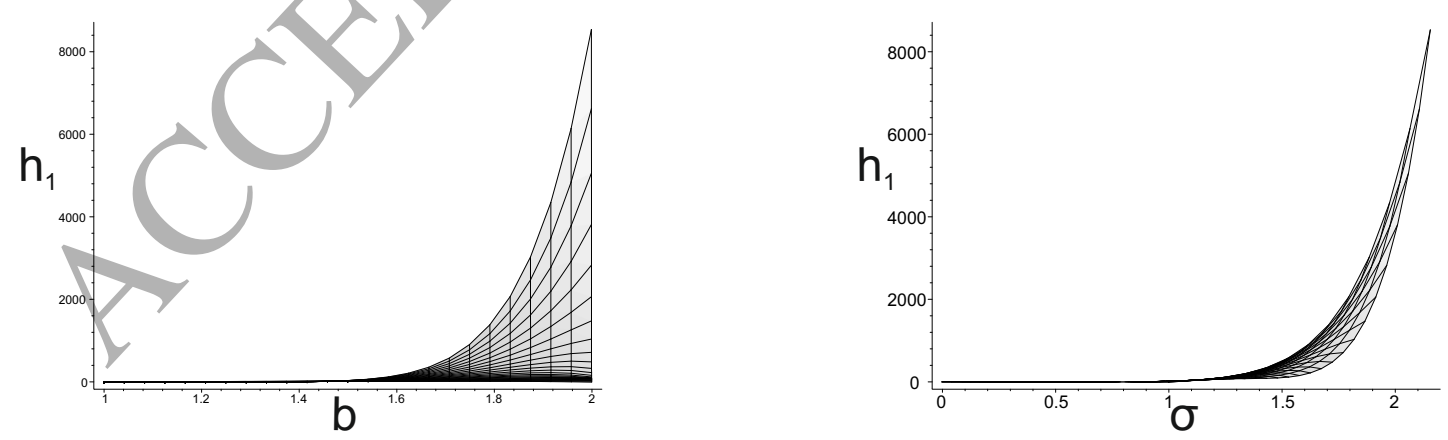

Figure 5: Projection of 3d plot of $h_{1}$ on the corresponding planes

VI. If $\sigma \leq 1,1<b \leq 2$, then the inequality $2 \sigma-b<0$ is valid. In this case the inequality $2 \sigma-b<0$ is a sufficient condition for (60). So if $\sigma \leq 1$, we take $\sigma>\frac{b}{2}$. 
Since we let $\sigma<1$, in condition (60) $\lambda_{0}=\sigma$. Then we obtain the following sufficient condition (consider $\lambda=\sigma$ in the right-hand side of inequality):

$$
2 \sigma-b<\frac{(\sigma+1)(\sigma+r)(b-\sigma)}{r^{2}}
$$

which is transformed to the following quadratic inequality with respect to $r$

$$
(2 \sigma-\sigma b) r^{2}+\left(\sigma^{3}-\sigma^{2} b-\sigma b+\sigma^{2}\right) r+\sigma^{4}-\sigma^{3} b-\sigma^{2} b+\sigma^{3}<0 .
$$

It is easy to check that the roots of the corresponding quadratic equation

$$
\begin{aligned}
& r^{(1)}=\frac{\sigma b-\sigma^{2}+b-\sigma-\sqrt{-(\sigma+1)(b-\sigma)((3 \sigma-1) b-(7 \sigma-1) \sigma)}}{2(2 \sigma-b)} \\
& r^{(2)}=\frac{\sigma b-\sigma^{2}+b-\sigma+\sqrt{-(\sigma+1)(b-\sigma)((3 \sigma-1) b-(7 \sigma-1) \sigma)}}{2(2 \sigma-b)}
\end{aligned}
$$

exist and $r^{(1)}<0<r^{(2)}$ if $\sigma<1$ and $1<b \leq 2$. Since in $(60)$ the coefficient of $r^{2}$ is positive provided that $\sigma$ and $b$ are from the considered domain, then we need to consider the relation $0<r-1<r^{(2)}-1$.

If the inequality

$$
\begin{gathered}
\frac{b(b+\sigma-1)^{2}-4 \sigma\left(\sigma b+b-b^{2}\right)}{\sigma^{2}(4-b)} \Varangle \\
<\frac{\sigma b-\sigma^{2}+b-\sigma+\sqrt{-(\sigma+1)(b-\sigma)((3 \sigma-1) b-(7 \sigma-1) \sigma)}}{2(2 \sigma-b)}-1
\end{gathered}
$$

is valid, then Theorem 3 is hold for $\sigma \in\left(\frac{b}{2}, \min \left(\sigma_{2}, 1\right)\right), b \in(1,2], r-1>0$.

Since we consider $\sigma \in\left(\frac{b}{2}, \min \left(\sigma_{2}, 1\right)\right), b \in(1,2]$, we have $\sigma^{2}(4-b)(2 \sigma-b)>0$ and inequality (64) is transformed as

$$
\begin{gathered}
(4-b) \sigma^{4}+\left(b^{2}-21 b+20\right) \sigma^{3}\left(33 b^{2}-36 b\right) \sigma^{2}+\left(-8 b^{3}+4 b^{2}+4 b\right) \sigma-2 b^{4}+4 b^{3}-2 b^{2}< \\
<\sigma^{2}(4-b) \sqrt{-(\sigma+1)(b-\sigma)((3 \sigma-1) b-(7 \sigma-1) \sigma)} .
\end{gathered}
$$

If the left-hand side of (65) is positive, then both sides can be squared:

$$
\begin{aligned}
&\left((4-b) \sigma^{4}+\left(b^{2}-21 b+20\right) \sigma^{3}\left(33 b^{2}-36 b\right) \sigma^{2}+\left(-8 b^{3}+4 b^{2}+4 b\right) \sigma-2 b^{4}+4 b^{3}-2 b^{2}\right)^{2}< \\
&<-\sigma^{4}(4-b)^{2}(\sigma+1)(b-\sigma)((3 \sigma-1) b-(7 \sigma-1) \sigma) \\
& \Leftrightarrow \\
& 0<-8(b-4)^{2} \sigma^{8}+\left(12 b^{3}-136 b^{2}+416 b-256\right) \sigma^{7}+\left(-4 b^{4}+140 b^{3}-928 b^{2}+1248 b-384\right) \sigma^{6}+ \\
&+\left(-84 b^{4}+1544 b^{3}-2872 b^{2}+1376 b\right) \sigma^{5}+\left(12 b^{5}-1408 b^{4}+2812 b^{3}-1256 b^{2}-160 b\right) \sigma^{4}+ \\
&+\left(4 b^{6}+436 b^{5}-588 b^{4}-220 b^{3}+368 b^{2}\right) \sigma^{3}+\left(68 b^{6}-344 b^{5}+468 b^{4}-176 b^{3}-16 b^{2}\right) \sigma^{2}+ \\
&+\left(-32 b^{7}+80 b^{6}-48 b^{5}-16 b^{4}+16 b^{3}\right) \sigma-4 b^{8}+16 b^{7}-24 b^{6}+16 b^{5}-4 b^{4}=: h_{2}(\sigma, b)=h_{2} .
\end{aligned}
$$

As is shown in Fig. 6, inequality (66) is valid. So inequality (64) is satisfied.

Thus, Theorem 3 is hold if $1<b \leq 2, r>1$.

\subsection{Proof of Corollary 4}

I. We consider the following sufficient condition for the theorem to be valid:

$$
\left\{\begin{array}{l}
r-1>\frac{b(b+\sigma-1)^{2}-4 \sigma\left(b+\sigma b-b^{2}\right)}{3 \sigma^{2}} \\
\sigma^{2}(r-1)(b-4)<4 \sigma\left(\sigma b+b-b^{2}\right)-b(b+\sigma-1)^{2}
\end{array}\right.
$$



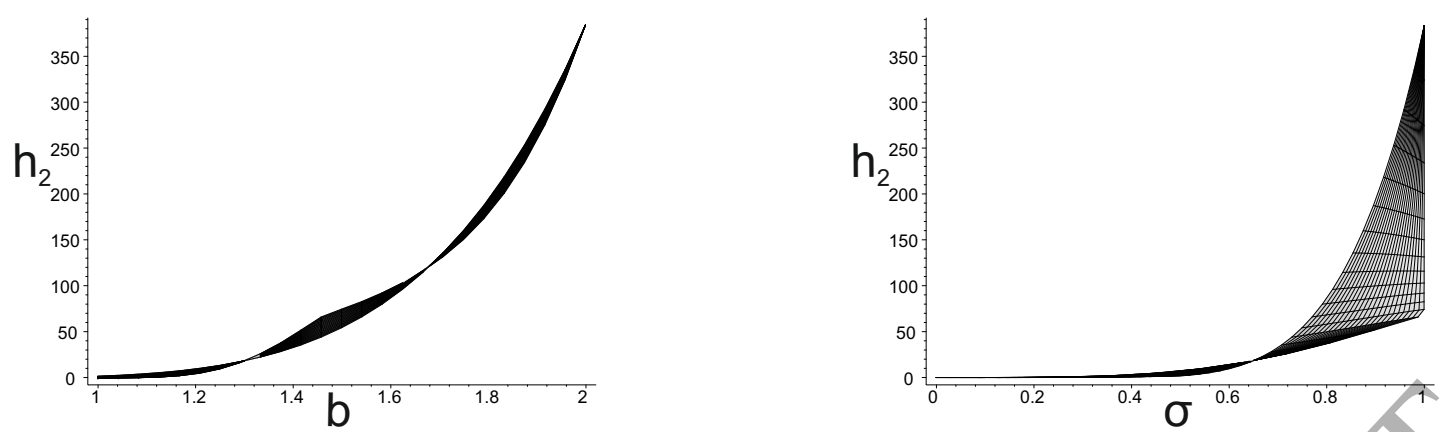

Figure 6: Projection of $3 \mathrm{~d}$ plot of $h_{2}$ on the corresponding planes.

and show that this condition is true for sufficiently large $\sigma$. The domain in which condition (67) is not satisfied is bounded with respect to $\sigma$. Since we consider $b<4$ and $\sigma>0$, system (67) is equivalent to the following one

$$
\begin{gathered}
\left\{\begin{array}{l}
r-1>\frac{b(b+\sigma-1)^{2}-4 \sigma\left(b+\sigma b-b^{2}\right)}{3 \sigma^{2}} \\
r-1>\frac{b(b+\sigma-1)^{2}-4 \sigma\left(b+\sigma b-b^{2}\right)}{\sigma^{2}(4-b)}
\end{array}\right. \\
\left\{\begin{array}{l}
\Leftrightarrow \\
r-1>\frac{b\left(\frac{b}{\sigma}+1-\frac{1}{\sigma}\right)^{2}-4\left(\frac{b}{\sigma}+b-\frac{b^{2}}{\sigma}\right)}{3} \\
r-1>\frac{b\left(\frac{b}{\sigma}+1-\frac{1}{\sigma}\right)^{2}-4\left(\frac{b}{\sigma}+b-\frac{b^{2}}{\sigma}\right)}{4-b}
\end{array}\right.
\end{gathered}
$$

Let

$$
\begin{aligned}
f(b, \sigma) & =\left(\frac{b}{\sigma}+1-\frac{1}{\sigma}\right)^{2}-4\left(\frac{1}{\sigma}+1-\frac{b}{\sigma}\right)= \\
& =\frac{1}{\sigma^{2}} b^{2}+\frac{-2+6 \sigma}{\sigma^{2}} b+\frac{-6 \sigma+1-3 \sigma^{2}}{\sigma^{2}}
\end{aligned}
$$

Then system (68) can be represented as

$$
\left\{\begin{array}{l}
r-1>\frac{b}{3} f(b, \sigma) \\
r-1>\frac{b}{4-b} f(b, \sigma)
\end{array}\right.
$$

The function $f(b, \sigma)$ is quadratic polynomial in $b$ with a positive coefficient of $b^{2}$. The abscissa of parabola peak is equal to $b_{0}=1-3 \sigma$. If we consider $\sigma>\frac{1}{3}$, then $b_{0}<0$ and, therefore, on the interval $b \in(0,4)$ the maximum of the function $f(b, \sigma)$ is achieved for $b=4$ and has the form

$$
f(4, \sigma)=-3+\frac{18}{\sigma}+\frac{9}{\sigma^{2}} \text {. }
$$


For $\sigma>3+\sqrt{12}$ we have $f(4, \sigma)<0$.

Since $b \in(0,4)$ and $r>1$, for sufficiently large $\sigma$ we get

$$
\left\{\begin{array}{l}
r-1>0>\frac{b}{3} f(b, \sigma) \\
r-1>0>\frac{b}{4-b} f(b, \sigma),
\end{array}\right.
$$

i.e. condition (67) is satisfied.

II. Let us show that for sufficiently large $\sigma$ and $r>1$ the inequality

$$
\sigma r>(b-\sigma)(b-1)
$$

is satisfied. Then the domain in which it is not satisfied is bounded with respect to $\sigma$.

Condition (73) is equivalent to

$$
r>\frac{1}{\sigma} b^{2}+\frac{-\sigma-1}{\sigma} b+1
$$

The right-hand side of this inequality is quadratic polynomial in $b$ with a positive coefficient of $b^{2}$. The abscissa of parabola peak is equal to $b_{0}^{\prime}=\frac{\sigma}{2}+\frac{1}{2}$. If $\sigma>7$, then $b_{0}^{\prime}>4$. In this case the maximum of the right-hand side of the inequality for $b \in(0,4)$ is smaller than that for $b=0$, i.e. 1. Thus, for sufficiently large $\sigma, b \in(0,4)$ and $r>1$ the relation

$$
r>1>\frac{1}{\sigma} b^{2}+\frac{-\sigma-1}{\sigma} b+1
$$

is satisfied, i.e. (73) is valid.

III. If $r>1, b \in(0,4)$, and $\sigma>7$, then condition (67) and inequality (73) are valid, i.e. the conditions of Theorem 3 are satisfied.

\section{Conclusion}

In this work we demonstrated that a previously known result for the Lyapunov dimension of the Lorenz system holds true for a larger set of system parameters. In particular, it is shown that the Lyapunov dimension formula may be valid in the case of one unstable and two stable equilibria. Also by analytical and numerical methods we have checked that the Lyapunov dimension formula obtained is valid for $0<b<4$.

\section{Acknowledgements}

This work was supported by the Russian Scientific Foundation (project 14-21-00041) and SaintPetersburg State University.

\section{References}

[1] Boichenko, V. A., Leonov, G. A., and Reitmann, V. (2005). Dimension Theory for Ordinary Differential Equations. Teubner, Stuttgart.

[2] Brezetskyi, S., Dudkowski, D., and Kapitaniak, T. (2015). Rare and hidden attractors in van der Pol-Duffing oscillators. European Physical Journal: Special Topics, 224(8):1459-1467. 
[3] Celikovsky, S. and Vanecek, A. (1994). Bilinear systems and chaos. Kybernetika, 30:403-424.

[4] Chen, G. and Ueta, T. (1999). Yet another chaotic attractor. International Journal of Bifurcation and Chaos, 9(7):1465-1466.

[5] Chueshov, I. (2002). Introduction to the Theory of Infinite-dimensional Dissipative Systems. Electronic library of mathematics. ACTA.

[6] Constantin, P., Foias, C., and Temam, R. (1985). Attractors representing turbulent flows. Memoirs of the American Mathematical Society, 53(314).

[7] Doering, C. R. and Gibbon, J. (1995). On the shape and dimension of the Lorenz attractor. Dynamics and Stability of Systems, 10(3):255-268.

[8] Douady, A. and Oesterle, J. (1980). Dimension de Hausdorff des attracteurs. C.R. Acad. Sci. Paris, Ser. A. (in French), 290(24):1135-1138.

[9] Eden, A. (1989a). An abstract theory of L-exponents with applications to dimension analysis (PhD thesis). Indiana University.

[10] Eden, A. (1989b). Local Lyapunov exponents and a local estimate of Hausdorff dimension. ESAIM: Mathematical Modelling and Numerical Analysis - Modelisation Mathematique et Analyse Numerique, 23(3):405-413.

[11] Eden, A. (1990). Local estimates for the Hausdorff dimension of an attractor. Journal of Mathematical Analysis and Applications, 150(1):100-119.

[12] Eden, A., Foias, C., and Temam, R. (1991). Local and global Lyapunov exponents. Journal of Dynamics and Differential Equations, 3(1):133-177. [Preprint No. 8804, The Institute for Applied Mathematics and Scientific Computing, Indiana University, 1988].

[13] Feng, Y., Pu, J., and Wei, Z. (2015). Switched generalized function projective synchronization of two hyperchaotic systems with hidden attractors. European Physical Journal: Special Topics, 224(8):1593-1604.

[14] Feng, Y. and Wei, Z. (2015). Delayed feedback control and bifurcation analysis of the generalized Sprott B system with hidden attractors. European Physical Journal: Special Topics, 224(8):1619-1636.

[15] Jafari, S., Sprott, J., and Nazarimehr, F. (2015). Recent new examples of hidden attractors. European Physical Journal: Special Topics, 224(8):1469-1476.

[16] Kaplan, J. L. and Yorke, J. A. (1979). Chaotic behavior of multidimensional difference equations. In Functional Differential Equations and Approximations of Fixed Points, pages 204-227. Springer, Berlin.

[17] Kuzhetsov, N. (2016a). Hidden attractors in fundamental problems and engineering models. A short survey. Lecture Notes in Electrical Engineering, 371:13-25. (Plenary lecture at AETA 2015: Recent Advances in Electrical Engineering and Related Sciences).

[18] Kuznetsov, N. (2016b). The Lyapunov dimension and its estimation via the Leonov method. Physics Letters A. (http://dx.doi.org/10.1016/j.physleta.2016.04.036). 
[19] Kuznetsov, N., Alexeeva, T., and Leonov, G. (2016). Invariance of Lyapunov exponents and Lyapunov dimension for regular and irregular linearizations. Nonlinear Dynamics, pages 1-7. (http://dx.doi.org/10.1007/s11071-016-2678-4).

[20] Kuznetsov, N., Leonov, G., and Mokaev, T. (2015). Hidden attractor in the Rabinovich system. arXiv:1504.04723v1. http://arxiv.org/pdf/1504.04723v1.pdf.

[21] Kuznetsov, N., Leonov, G., and Vagaitsev, V. (2010). Analytical-numerical method for attractor localization of generalized Chua's system. IFAC Proceedings Volumes (IFAC-PapersOnline), 4(1):29-33.

[22] Kuznetsov, N., Mokaev, T., and Vasilyev, P. (2014). Numerical justification of Leonov conjecture on Lyapunov dimension of Rossler attractor. Commun Nonlinear Sci Numer Simulat, 19:1027-1034.

[23] Leonov, G. (2002). Lyapunov dimension formulas for Henon and Lorenz attractors. St.Petersburg Mathematical Journal, 13(3):453-464.

[24] Leonov, G. (2013). Formulas for the Lyapunov dimension of attractors of the generalized Lorenz system. Doklady Mathematics, 87(3):264-268.

[25] Leonov, G., Alexeeva, T., and Kuznetsov, N. (2015a). Analytic exact upper bound for the Lyapunov dimension of the Shimizu-Morioka system. Entropy. 17(7):5101-5116.

[26] Leonov, G. and Kuznetsov, N. (2013). Hidden attractors in dynamical systems. From hidden oscillations in Hilbert-Kolmogorov, Aizerman, and Kalman problems to hidden chaotic attractors in Chua circuits. International Journal of Bifurcation and Chaos, 23(1). art. no. 1330002.

[27] Leonov, G. and Kuznetsov, N. (2015), On differences and similarities in the analysis of Lorenz, Chen, and Lu systems. Applied Mathematics and Computation, 256:334-343.

[28] Leonov, G., Kuznetsov, N., Korzhemanova, N., and Kusakin, D. (2015b). Estimation of Lyapunov dimension for the Chen and Lusystems. arXiv, http://arxiv.org/pdf/1504.04726v1.pdf.

[29] Leonov, G., Kuznetsov, N., Korzhemanova, N., and Kusakin, D. (2015d). The Lyapunov dimension formula for the global attractor of the Lorenz system. arXiv, http://arxiv.org/pdf/1508.07498v1.pdf.

[30] Leonov, G., Kuznetsov, N., Korzhemanova, N., and Kusakin, D. (2015c). Lyapunov dimension formula of attractors in the Tigan and Yang systems. arXiv:1510.01492v1, http://arxiv.org/pdf/1510.01492v1.pdf.

[31] Leonov, G., Kuznetsov, N., and Mokaev, T. (2015e). Hidden attractor and homoclinic orbit in Lorenz-like system describing convective fluid motion in rotating cavity. Communications in Nonlinear Science and Numerical Simulation, 28:166-174.

[32] Leonov, G., Kuznetsov, N., and Mokaev, T. (2015f). Homoclinic orbits, and self-excited and hidden attractors in a Lorenz-like system describing convective fluid motion. Eur. Phys. J. Special Topics, 224(8):1421-1458.

[33] Leonov, G., Kuznetsov, N., and Mokaev, T. (2015g). The Lyapunov dimension formula of self-excited and hidden attractors in the Glukhovsky-Dolzhansky system. arXiv:1509.09161. http://arxiv.org/pdf/1509.09161v1.pdf. 
[34] Leonov, G., Kuznetsov, N., and Vagaitsev, V. (2011). Localization of hidden Chua's attractors. Physics Letters A, 375(23):2230-2233.

[35] Leonov, G., Kuznetsov, N., and Vagaitsev, V. (2012a). Hidden attractor in smooth Chua systems. Physica D: Nonlinear Phenomena, 241(18):1482-1486.

[36] Leonov, G. and Lyashko, S. (1993). Eden's hypothesis for a Lorenz system. Vestnik St. Petersburg University: Mathematics, 26(3):15-18. [Transl. from Russian: Vestnik SanktPeterburgskogo Universiteta. Ser 1. Matematika, 26(3), 14-16].

[37] Leonov, G., Pogromsky, A., and Starkov, K. (2012b). Erratum to "The dimension formula for the Lorenz attractor" [Phys. Lett. A 375 (8) (2011) 1179]. Physics Letters A, 376(45):3472 -3474 .

[38] Leonov, G. and Poltinnikova, M. (2005). On the Lyapunov dimension of the attractor of Chirikov dissipative mapping. AMS Translations. Proceedings of St.Petersburg Mathematical Society. Vol. X, 224:15-28.

[39] Leonov, G. A. (1983). On the global stability of the Lorenz system. Journal of Applied Mathematics and Mechanics, 47(5):861-863.

[40] Leonov, G. A. (1991). On estimations of Hausdorff dimension of attractors. Vestnik St. Petersburg University: Mathematics, 24(3):38-41. [Transl. from Russian: Vestnik Leningradskogo Universiteta. Mathematika, 24(3), 1991, pp. 41-44].

[41] Leonov, G. A. (2008). Strange attractors and classical stability theory. St.Petersburg University Press, St.Petersburg.

[42] Leonov, G. A. (2012). Lyapunov functions in the attractors dimension theory. Journal of Applied Mathematics and Mechanics, 76(2):129-141.

[43] Leonov, G. A. and Boichenko, V. A. (1992). Lyapunov's direct method in the estimation of the Hausdorff dimension of attractors. Acta Applicandae Mathematicae, 26(1):1-60.

[44] Leonov, G. A., Bunin, A. I., and Koksch, N. (1987). Attraktorlokalisierung des Lorenzsystems. ZAMM - Journal of Applied Mathematics and Mechanics / Zeitschrift fur Angewandte Mathematik und Mechanik, 67(12):649-656.

[45] Li, C., Hu, W., Sprott, J., and Wang, X. (2015). Multistability in symmetric chaotic systems. European Physical Journal: Special Topics, 224(8):1493-1506.

[46] Lorenz, E. N. (1963). Deterministic nonperiodic flow. J. Atmos. Sci., 20(2):130-141.

[47] Lu, J. and Chen, G. (2002). A new chaotic attractor coined. Int. J. Bifurcation and Chaos, 12:1789-1812.

[48] Pham, V., Vaidyanathan, S., Volos, C., and Jafari, S. (2015). Hidden attractors in a chaotic system with an exponential nonlinear term. European Physical Journal: Special Topics, 224(8):1507-1517.

[49] Saha, P., Saha, D., Ray, A., and Chowdhury, A. (2015). Memristive non-linear system and hidden attractor. European Physical Journal: Special Topics, 224(8):1563-1574. 
[50] Semenov, V., Korneev, I., Arinushkin, P., Strelkova, G., Vadivasova, T., and Anishchenko, V. (2015). Numerical and experimental studies of attractors in memristor-based Chua's oscillator with a line of equilibria. Noise-induced effects. European Physical Journal: Special Topics, 224(8):1553-1561.

[51] Shahzad, M., Pham, V.-T., Ahmad, M., Jafari, S., and Hadaeghi, F. (2015). Synchronization and circuit design of a chaotic system with coexisting hidden attractors. European Physical Journal: Special Topics, 224(8):1637-1652.

[52] Sharma, P., Shrimali, M., Prasad, A., Kuznetsov, N., and Leonov, G. (2015). Control of multistability in hidden attractors. Eur. Phys. J. Special Topics, 224(8):1485-1491.

[53] Sparrow, C. (1982). The Lorenz Equations: Bifurcations, Chaos, and Strange Attractors. Applied Mathematical Sciences. Springer New York.

[54] Sprott, J. (2015). Strange attractors with various equilibrium types. European Physical Journal: Special Topics, 224(8):1409-1419.

[55] Tigan, G. and Opris, D. (2008). Analysis of a 3d chaotic system. Chalos, Solitons E Fractals, 36(5):1315-1319.

[56] Vaidyanathan, S., Pham, V.-T., and Volos, C. (2015), A 5-D hyperchaotic Rikitake dynamo system with hidden attractors. European Physical Journal: Special Topics, 224(8):1575-1592.

[57] Yu, P. and Chen, G. (2004). Hopf bifurcation control using nonlinear feedback with polynomial functions. International Journal of Bifurcation and Chaos, 14(05):1683-1704.

[58] Zhusubaliyev, Z., Mosekilde, E., Churilov, A., and Medvedev, A. (2015). Multistability and hidden attractors in an impulsive Goodwin oscillator with time delay. European Physical Journal: Special Topics, 224(8):1519-1539. 